\title{
Emergence of jams in the generalized totally asymmetric simple exclusion process
}

\author{
A.E. Derbyshev' \\ Bogoliubov Laboratory of Theoretical Physics, Joint Institute for Nuclear Research, Dubna, Russia and \\ Moscow Institute of Physics and Technology, Dolgoprudny, Russia \\ A.M.Povolotsky ${ }^{\dagger}$ \\ Bogoliubov Laboratory of Theoretical Physics, Joint Institute for Nuclear Research, 141980 Dubna, Russia and \\ Laboratory of Mathematical Physics, NRU HSE, Moscow, Russia \\ V. B. Priezzhev \\ Bogoliubov Laboratory of Theoretical Physics, Joint Institute for Nuclear Research, 141980 Dubna, Russia
}

\begin{abstract}
The generalized totally asymmetric exclusion process (TASEP) [J. Stat. Mech. P05014 (2012)] is an integrable generalization of the TASEP equipped with an interaction, which enhances the clustering of particles. The process interpolates between two extremal cases: the TASEP with parallel update and the process with all particles irreversibly merging into a single cluster moving as an isolated particle. We are interested in the large time behavior of this process on a ring in the whole range of the parameter $\lambda$ controlling the interaction. We study the stationary state correlations, the cluster size distribution and the large-time fluctuations of integrated particle current. When $\lambda$ is finite, we find the usual TASEP-like behavior: The correlation length is finite; there are only clusters of finite size in the stationary state and current fluctuations belong to the Kardar-ParisiZhang universality class. When $\lambda$ grows with the system size so does the correlation length. We find a nontrivial transition regime with clusters of all sizes on the lattice. We identify a crossover parameter and derive the large deviation function for particle current, which interpolates between the case considered by Derrida-Lebowitz and a single particle diffusion.
\end{abstract}

PACS numbers: 74.40.Gh,02.30.Ik

\section{INTRODUCTION}

The asymmetric simple exclusion process (ASEP) is one of the basic models of driven transport admitting an analytical treatment 1 - 3 . It is commonly accepted that different versions of ASEP provide an adequate description of statistical properties of one-dimensional diffusive and driven-diffusive systems. During the last decades the ASEP was a laboratory for obtaining the universal critical exponents and scaling functions of the EdwardWilkinson (EW) and Kardar-Parisi-Zhang (KPZ) universality classes [4, 5]. The range of models that can be solved exactly is very limited, but the universality implies that results obtained from their solution apply to a wide range of stochastic systems, like interacting particle systems, growing interfaces, crystal facets, polymers in random media, etc. Among the results, which are believed to be universal, are the dynamical exponent of the KPZ class [6], the KPZ-EW crossover function for the relaxation time [7, the large deviation function (LDF) for particle current in the systems with periodic [8] and open [9, 10] boundary conditions. More recently, consideration of these processes on the infinite lattice yielded plenty of results on the universal scaling functions for probability

\footnotetext{
*Electronic address: derbishev@theor.jinr.ru

†Electronic address: alexander.povolotsky@gmail.com

${ }_{\ddagger}$ Electronic address: priezzvb@theor.jinr.ru
}

distributions and correlation functions characterizing the nonstationary time evolution.

The totally asymmetric exclusion process (TASEP) is the simplest version of the ASEP, possessing a special mathematical structure, which simplifies the solution significantly. Using this structure, Derrida and Lebowitz obtained the first exact expression for the LDF of particle current for an arbitrary lattice size, which yielded the universal scaling function in the scaling limit. Also, closed determinantal formulas for the Green's functions were derived for the TASEP on both the infinite lattice [11] and the ring [12]. Finally, all multipoint correlation functions for the process in the infinite system were constructed [13 20]. Remarkably, unlike the partially asymmetric case, the TASEP remains exactly solvable in a discrete time framework. The models with several different updates were solved: backward sequential [21, parallel 22] and sublattice parallel [23]. All these versions of the TASEP demonstrate the same universal KPZ behavior in the scaling limit. It is of interest, however, to examine possible mechanisms taking the system away from the KPZ class, to see how the KPZ universality breaks down.

To our knowledge, the generalized TASEP (gTASEP) studied here was first considered in [24], where without any reference to its integrable structure, it was used as an example of a traffic model with a stationary measure admitting factorized representation. It was later rediscovered in [25], within a totally different context as an integrable generalization of the TASEP. Finally, it was shown to be a particular $q=0$ limiting case of the 
general three parametric Bethe ansatz-solvable stochastic chipping model [26], also referred to as a q-Hahn or $(q, \mu, \nu)$-boson process [27, 28]. In turn, it containes already known TASEPs with parallel and sequential update as particular cases. In the gTASEP an additional interaction between particles is introduced, which enhances the clustering of particles comparing to the usual TASEP. The dynamics of the model can be viewed as the TASEPlike process, where clusters of particles diffuse, breaking into parts and merging together. The relative frequency of these processes is controlled by an extra parameter $\lambda$. The bigger value of $\lambda$, the stronger is the effective attraction between particles and the larger is the size of clusters in typical particle configuration. A limiting case $\lambda \rightarrow \infty$, which we refer to as the deterministic aggregation (DA) limit, produces the process, where particles stick together irreversibly, finally forming a single giant cluster, which moves as an ordinary random walk.

The main aim of the present paper is to study how the large-scale behavior of the steady state in gTASEP changes as the DA limit is approached. We concentrate on the stationary state correlations and fluctuations of particle current on the ring. For moderate interaction strength it is natural to expect that the scaling behavior of gTASEP will be similar to the usual TASEP, which belongs to the KPZ universality class. For the latter, it is well known that the stationary state is uncorrelated if looked at in the scale of the system size. Also the motion of particles in an infinite system is subdiffusive. Though it is still diffusive in a finite system, the diffusion coefficient decays as $\Delta \sim 1 / \sqrt{L}$ as the system size $L$ grows to infinity. Further details of the large time fluctuations of particle current can be extracted from the universal LDF obtained by Derrida and Lebowitz in [8] for the usual TASEP, and later proved to hold for several other systems 2932 . On the other hand in the DA limit the particles form a single giant cluster, which moves as a single particle. This behavior obviously corresponds to correlation length unboundedly growing with the system size and to purely diffusive motion of each individual particle. As a result, there are many small particle clusters, finite range correlations, and KPZ-like fluctuations on the one end of the range of $\lambda$ and one macroscopic cluster with pure single-particle diffusion on the other. Then the natural question to ask is how many particle clusters can there be and how large can typical particle clusters be for the KPZ universality to survive and how the two regimes are connected to each other. Intuition says that at least at a finite density of finite clusters, which is maintained at finite values of $\lambda$, we should be in the KPZ regime, as the finite clusters can be effectively treated as larger particles. The analysis below shows, however, that one can approach the DA limit much more closely keeping the universal KPZ form of the current LDF. We show that even when $\lambda$ and, hence, the typical size of clusters, grow with the system size, the LDF preserves its functional form, unless the order of $\lambda$ is as large as $L^{2}$. When $\lambda / L^{2} \rightarrow 0$, the dependence on the value of $\lambda$ affects only the non-universal constants controlling the fluctuation scale but not the functional form of the distribution. At the scale $\lambda \sim L^{2}$ there is only a few (a finite number of) macroscopic clusters on the lattice and the correlation length is of order of system size $L$. At this scale, the transition from the KPZ to the DA limit takes place. We obtain the LDF that crosses over from the KPZ Derrida-Lebowitz form to pure Gaussian as $\lambda / L^{2}$ varies from zero to infinity.

To have a rough idea of where the scale $\lambda \sim L^{2}$ comes from, the following simple mean-field argument can be used, which should not be considered as a derivation, but can be viewed as a description of the scenario of the transtion regime. Let us think about the large number $M$ of interacting particles diffusing on the one-dimensional lattice with overall particle density being fixed, $M / L=c$, as about diffusing, aggregating and dissociating clusters (compact groups of particles). Two clusters merge when coming in contact, while any cluster can break down into two smaller clusters at any point with small rate $\alpha$. Then as $\alpha$ goes to zero, we expect to observe a transition from a finite density of finite clusters to a single cluster of size $M$. In the transition regime there is a finite number of clusters of any macroscopic size. Under this suggestion consider the conditions for the equilibrium to hold between merging and breaking up clusters at all scales. Let $P(n)$ be a global density (mean number per unit length) of $n$-particle clusters, which is supposed to be of the same order through the whole range of $n$ in the transition regime. The number of clusters of size $n$ in the system is equal to $L P(n)$, and the total number of clusters of any size in a typical configuration is given by the sum $L \sum_{1 \leq n \leq M} P(n)$. For this number to be finite (of order of one), the value of $P(n)$ should be of order of $1 / L^{2}$. As the cluster of $n$ particles can split into two smaller clusters at any of its points, the mean rate of decay of such clusters will be $n \alpha P(n)$. On the other hand, the number of clusters of size $n$ appearing per unit time is $\sum_{k} P(k) P(n-k)$, which is of order of $1 / L^{3}$. Equating these two expressions we find that $\alpha$ must be of order of $1 / L^{2}$. An analog of splitting rate $\alpha$ in our model is the inverse of the parameter $\lambda$. Our asymptotic analysis indeed shows that the scaling parameter controlling the transition occurring in the limit $\lambda \rightarrow \infty$ can be chosen proportional to $\lambda / L^{2}$. Tuning this parameter one can obtain both the particle current LDFs for KPZ and DA regimes as limiting cases. We want to emphasize that the above description gives a qualitative picture, which can only illustrate the exact results obtained below.

It is worth mentioning other studies of models, where the particle clustering strength can be controlled. A version of the TASEP with next-nearest neighbor interaction was proposed in 33. The particle flow has the jamming tendency and for this reason the flow diagram is shifted in the region of large densities. The finding of a "fourth phase" in the mean-field approximation (approved by Monte Carlo simulations) demonstrates an unusual and nontrivial character of particle flow when it 
enters the jam regime.

The model which allows for the diffusion of clusters, aggregation on contacts between them and single-particle dissociation has been considered in 34. A mean field analysis of the model showed that the system undergoes the dynamical phase transition: The steady state mass distribution in one phase decays exponentially for large masses. In another phase, the model predicts an infinite aggregate in addition to a power-law mass decay.

Note that the mentioned models do not belong to the class of integrable models. The models like these are generally studied in the mean field approximation, or at best allow the exact characterization of the stationary state distribution; see, e.g., 33. Such an analysis provides the thermodynamical description, like the densitycurrent relation, which is not universal and to large extent depends on particular dynamical rules. In contrast, in our case the integrability allows the exact treatment of the full dynamical problem, which contains information about universal fluctuations in the scaling limit.

Our paper is organized as follows. In Sec. III we formulate the model and explain the zero-ramge rocess (ZRP)ASEP mapping, which allows us to establish a relation between gTASEP and another zero-range type model with an unbounded number of particles in a site. While many quantities characterizing the two models coincide, the advantage of models like the ZRP is a factorized form of steady state distribution, which can be analyzed with the canonical partition function formalism.

In Sec. III we study the stationary state of both gTASEP and the corresponding ZRP-like model. For the ZRP-like model we obtain the exact expressions for the partition function on an arbitrary finite lattice and use it to derive the occupation number distribution. The latter can be reinterpreted as the cluster size distribution in the gTASEP. We also derive the generating function of particle jumps and, in particular, obtain the exact formula for the mean number of particle jumps per unit time. The exact partition function and particle current are represented as contour integrals, which, then, are explicitly evaluated in terms of the Gauss ${ }_{2} F_{1}$ and Appell $F_{1}$ terminating hypergeometric series, respectively. Then we perform an asymptotic analysis of the integrals obtained, first, in the saddle point approximation, which is applicable when $\lambda / L^{2} \rightarrow 0$, and, second, in the limit $\lambda / L^{2} \rightarrow$ const $>0$, when the saddle point approximation fails. In the first case, we obtain the geometric finite (or subextensive) cluster size distribution and the thermodynamic formula for particle current (flow diagram) depending on two parameters and particle density. In the second case we obtain the distribution of cluster sizes on the system size scale, expressed in terms of the modified Bessel functions. In the last section of Sec. [II we analyze the stationary state of the gTASEP directly in the grand-canonical ensemble exploiting the fact that the stationary measure of the gTASEP is similar to the Gibbs measure of a one-dimensional Ising model. We evaluate the two-point correlation function and discuss its behav- ior in both limits.

Section IV] is devoted to the analysis of particle current fluctuations. We first remind the reader of the Bethe ansatz solution of the models discussed and then obtain the largest eigenvalue of the Markov matrix deformed by including parameter $\gamma$, counting the particle jumps. The eigenvalue, obtained in the parametric form as two series with coefficients expressed via ${ }_{2} F_{1}$ and $F_{1}$, has a meaning of the rescaled cumulant generating function of the total number of particle jumps or of the Legendre transform of the corresponding LDF. In particular, in addition to the exact particle current obtained in Sec. III we derive the exact expression for the diffusion coefficient of a particle in gTASEP. The asymptotic analysis again consists of two parts: the saddle point approximation for $\lambda / L^{2} \rightarrow 0$, which reproduces the universal function by Derrida and Lebowitz through a range of scales of $\lambda$, and the asymptotic analysis on the scale $\lambda \sim L^{2}$, describing the KPZ-to-Gauss transition.

The last section, Sec. V] is intended to bind together the variety of the results obtained for the KPZ regime. We remind to the reader of the scaling theory, developed in [35], which claims that many non-universal quantities characterizing the systems belonging to the KPZ universality class can be expressed in terms of only two dimensional invariants, which, in particular, are related to the dimensional constants in the KPZ equation. We show that this hypothesis is confirmed by our results and conversely express the non-universal scaling constants in the $\mathrm{LDF}$ in terms of the KPZ dimensional invariants.

In Appendix A we give explicit formulas for some model-dependent constants appearing in the calculations and establish relations between them, which confirm KPZ universality. The formulas used to work with the special functions are listed in Appendices B and C.

\section{MODEL DEFINITION AND ZRP-ASEP MAPPING}

Consider $M$ particles on the one-dimensional lattice of $L$ sites with periodic boundary conditions. Each lattice site can be occupied by, at most, one particle. Particle configurations are recorded as $N$-tuples of particle coordinates $\mathbf{x}=\left(1 \leq x_{1}<x_{2}<\cdots<x_{M} \leq L\right)$. Particle configurations evolve in discrete time with clusterwise backward-sequential update rules. We refer to a compact string of particles like $\left(x_{i-k}=x-k, \ldots, x_{i}=x\right)$ surrounded by two empty sites as a cluster. The update of particle configuration at a given time step starts from the rightmost particle of any cluster. For definiteness, one can choose the cluster with the maximal coordinate $x_{i} \leq L$ of the rightmost particle. The particle tries to jump one step to the right, i.e., clockwise, succeeding, $(x \rightarrow x+1 \bmod L)$, with probability $p$ or failing, $(x \rightarrow x)$, with probability $1-p$. In the case of success and if the cluster consists of more than one particle, the second particle tries to follow the first one with proba- 


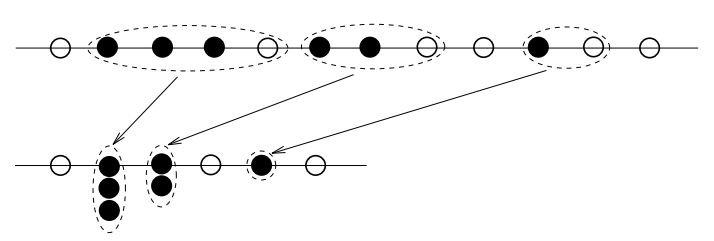

Figure 1: ZRP-ASEP mapping.

bility $\mu$, which is, in general, different from $p$. So do the third, fourth, etc., particles until either some particle of the cluster has failed to jump or the cluster has ended. In other words, for $k$-particle cluster with $k>1$ the following outcomes are possible:

1. all $k$ particles stay with probability $(1-p)$;

2. $m<k$ particles make a step with probability $p \mu^{m-1}(1-\mu)$

3. all $k$ particles jump with probability $p \mu^{k-1}$

Then we go to the next cluster in a counterclockwise direction and continue the update until all clusters on the lattice have been updated. Note that the result clearly does not depend on what cluster we choose to start. The clusterwise backward-sequential update, i.e. the condition of starting from the rightmost particle of a cluster excludes the situation when the tail of a cluster is updated before its head, which would occur with the conventional sitewise backward update, when the sites 1 and $L$ are inside the same cluster. It is also easy to see that the exclusion rule is automatically satisfied.

The above formulation uses two control parameters $p$ and $\mu$ having a meaning of probabilities, hence varying in the range $0 \leq p, \mu \leq 1$. The particular cases $\mu=0$ and $\mu=p$ correspond to the TASEP with parallel and backward-sequential updates, respectively. In the case $\mu=1$ the probability for all particles of a cluster to follow the first particle is equal to one. Therefore, clusters can only merge and no dissociation occurs in this limit.

Using so-called ZRP-ASEP mapping, the gTASEP can be related to a model of the ZRP type with an unbounded number of particles in a site. To establish the correspondence, we replace a string of sites occupied by a cluster of $n$ particles together with one empty side ahead by a single site with $n$ particles as shown in Fig. 1.

Thus, $M$ particles are placed on the lattice $\mathcal{L}$ consisting of $N=L-M$ sites allowed to hold any number of particles unlike at most one particle per site in the ASEP. The one-step jump of $m$ particles from a splitting cluster will be replaced with a jump of the same number of particles from the corresponding site to the next site on the right. According to the above dynamical rules, the jump of $m$ particles from a site with $n$ particles depends on both $n$ and $m$, and has the form

$$
\varphi(m \mid n)= \begin{cases}(1-p), & m=0 ; \\ p \mu^{m-1}(1-\mu), & 0<m<n ; \\ p \mu^{n-1} & m=n,\end{cases}
$$

for $n>0$ and $\varphi(0 \mid 0)=1$ with all the sites being updated simultaneously at a given time step, as in the parallel update scheme. As noted in Sec. II, the hopping probabilities of our model are a particular $q=0$ limit of the general three-parametric hopping probabilities of the model proposed in [26], where they depended on three parameters $q, \mu$ and $\nu$. Here we use the notations of [26], which are different from those used in the first paper on the gTASEP [25]. Specifically, the two parameters $\mu$ and $\nu$ of the present article (as well as of [26]) correspond to $p(1+\nu)$ and $\nu p /(1-p)$ of [25] respectively. The parameter $\nu$ used here is related to the parameters $p$ and $\mu$ defined above by

$$
\nu=\frac{\mu-p}{1-p} .
$$

In the following, where it is more appropriate for brevity of notations, we also use another parameter

$$
\lambda=\frac{1}{1-\nu} \text {. }
$$

In particular, it is convenient for studying the DA limit, which corresponds to $\lambda \rightarrow \infty$.

Note that for periodic lattices, the ZRP-ASEP mapping is not the one-to-one correspondence between particle configurations, though they can be made equivalent up to the lattice rotations. In particular, there are more particle configurations in ASEP-like systems that in ZRP-like systems. Given two initial particle configurations in the ZRP and the ASEP related to one another by the mapping, the further processes are in the one-to-one correspondence in terms of relative distances between particles, but not in terms of particle coordinates. The essence of the difference is different translational symmetries of the lattices with different numbers of sites: A particle configuration returns to itself after $L$ unit translations in the ASEP and after $M$ unit translations in the ZRP. As seen from the Bethe ansatz solution below, a little modification is necessary to transform formulas using the coordinate notations in one system to those in the other. However, this difference does not affect translation invariant quantities, e.g. stationary state observables such as particle density and current. Once calculated in one system such a quantity can be easily related to similar quantity in the other. The densities of particles defined in the TASEP-like and and ZRP-like systems as

$$
c=M / L \text { and } \rho=M / N,
$$

respectively, are related by

$$
c=\frac{\rho}{1+\rho} .
$$


The total numbers of jumps made by all particles are the same in both systems. In particular, this is the case for the stationary state average number of jumps per one step $J$. Then the mean velocities of particles $v=J / M$ are the same in both systems, while the stationary state currents, i.e., average numbers of particles leaving one site per time step, $j^{A S E P}=J / L$ and $J^{Z R P}=J / N$, satisfy the following relation:

$$
\frac{j^{A S E P}}{c}=\frac{j^{Z R P}}{\rho}=v .
$$

Below, we use these relations to express stationary characteristics of the gTASEP on terms of quantities obtained for corresponding ZRP-like system.

\section{STATIONARY STATE}

\section{A. Partition function formalism}

The advantage of the ZRP-like system is that its stationary measure has a particularly simple form. Specifically, consider the one-dimensional periodic lattice $\mathcal{L}=$ $\mathbb{Z} / N \mathbb{Z}$ consisting of $N$ sites with $M$ particles on it. Every site can hold any number of particles. It is convenient to specify particle configurations in the ZRP-like systems by $N$ occupation numbers of all sites

$$
\mathbf{n}=\left\{n_{1}, \ldots, n_{N}\right\} .
$$

The system configuration is updated at every time step by bringing any number $m_{i} \leq n_{i}$ of particles from every site $i=1, \ldots, N$ to the next site $i+1$ with probability $\varphi\left(m_{i} \mid n_{i}\right)$ defined in (1). Therefore, the probability $P_{t}(\mathbf{n})$ for the system to be in a configuration $\mathbf{n}$ at time step $t$ obeys the Markov equation

$$
P_{t+1}(\mathbf{n})=\sum_{\mathbf{n}^{\prime}} \mathbf{M}_{\mathbf{n}, \mathbf{n}^{\prime}} P_{t}\left(\mathbf{n}^{\prime}\right),
$$

with transition matrix $\mathbf{M}$ defined by matrix elements

$$
\mathbf{M}_{\mathbf{n}, \mathbf{n}^{\prime}}=\sum_{\left\{m_{k} \in \mathbb{Z}_{\geq 0}\right\}_{k \in \mathcal{L}}} \prod_{i \in \mathcal{L}} T_{n_{i}, n_{i}^{\prime}}^{m_{i-1}, m_{i}}
$$

where $T_{n_{i}, n_{i}^{\prime}}^{m_{i-1}, m_{i}}=\delta_{\left(n_{i}-n_{i}^{\prime}\right),\left(m_{i-1}-m_{i}\right)} \varphi\left(m_{i} \mid n_{i}^{\prime}\right)$. It was proved in [36] that if and only if the hopping probabilities $\varphi(m \mid n)$ have the functional form

$$
\varphi(m \mid n)=\frac{v(m) w(n-m)}{\sum_{i=0}^{n} v(i) w(n-i)},
$$

with two arbitrary positive functions $v(k)$ and $w(k)$, the Markov equation (5) has a unique stationary solution, which belongs to the class of the so-called product measures; i.e., the probability of a configuration is given by the product of one-site factors

$$
P_{s t}(\mathbf{n})=\frac{1}{Z(M, N)} \prod_{i=1}^{N} f\left(n_{i}\right)
$$

where the one-site factor is given by

$$
f(n)=\sum_{i=0}^{n} v(i) w(n-i),
$$

and $Z(M, N)$ is the normalization constant, referred to as the partition function in statistical physics. In our case, the functions $v(k)$ and $w(k)$ that define the hopping probabilities (1) can be chosen as

$$
\begin{aligned}
v(k) & =\mu^{k}\left(\delta_{k, 0}+\left(1-\delta_{k, 0}\right)(1-\nu / \mu)\right), \\
w(k) & =\left(\delta_{k, 0}+\left(1-\delta_{k, 0}\right)(1-\mu)\right),
\end{aligned}
$$

which, according to (9), yield an expression for the single site weight,

$$
f(n)=\left(\delta_{n, 0}+\left(1-\delta_{n, 0}\right)(1-\nu)\right)
$$

With the product measure (8) in hands, we are in position to use the partition function formalism [37, 38] to calculate the stationary state observables. The partition function is the normalization constant of the stationary distribution (8), given by the sum of unnormalized weights over all particle configurations,

$$
Z(M, N)=\sum_{n_{1}, \ldots, n_{N} \geq 0} \delta_{\|n\|, M} \prod_{i=1}^{N} f\left(n_{i}\right),
$$

Here $f(n)$ is the one-site weight defined in $12,\|n\|=$ $n_{1}+\cdots+n_{N}$, and the Kronecker $\delta$ symbol constrains the summation to particle configurations with the number of particles fixed to $M$. The sum is given by the contour integral

$$
Z(M, N)=\oint_{\Gamma_{0}} \frac{[F(z)]^{N}}{z^{M+1}} \frac{d z}{2 \pi i}
$$

where

$$
F(z)=\sum_{n=0}^{\infty} z^{n} f(n)
$$

is the generating function of the one-site weights, and the contour of integration is a small circle closed around the point $z=0$ leaving all other singularities of $F(z)$ outside. The partition function contains information about the stationary state of the model. In particular, the probability for a site to be occupied by $n$ particles is

$$
P(n)=f(n) \frac{Z(M-n, N-1)}{Z(M, N)} .
$$

Another correlation function is the probability $\mathcal{H}(k)$ for $k$ particles on the lattice to hop simultaneously,

$$
\mathcal{H}(k)=\sum_{\mathbf{n}, \mathbf{m} \in \mathbb{Z}_{\geq 0}^{N}} \delta_{\|\mathbf{m}\|, k} \delta_{\|\mathbf{n}\|, M} \varphi(\mathbf{m} \mid \mathbf{n}) P_{s t}(\mathbf{n}),
$$


where $\varphi(\mathbf{m} \mid \mathbf{n})=\prod_{1 \leq i \leq L} \varphi\left(m_{i} \mid n_{i}\right)$ and we suggest that $\varphi(m \mid n)=0$, when $m>n$. The corresponding generating function,

$$
\Psi(x) \equiv \sum_{n=0}^{M} x^{n} \mathcal{H}(n) .
$$

can be obtained in the form of contour integral

$$
\Psi(x)=\frac{1}{Z(M, N)} \oint_{\Gamma_{0}} \frac{[\Phi(x, z)]^{N}}{z^{M+1}} \frac{d z}{2 \pi i}
$$

with the two-variable generating function

$$
\Phi(x, z)=\sum_{n=0}^{\infty} \sum_{m=0}^{n} \varphi(m \mid n) f(n) x^{m} z^{n} .
$$

The values of $\mathcal{H}(n)$ can be then represented via contour integrals with $\Psi(x)$ around $x=0$, while for the moments we need the derivatives at $x=1$. In particular, the average total number of particles jumping per unit time can be evaluated as

$$
J=\Psi^{\prime}(1)
$$

Using the representation (7) of $\varphi(m \mid n)$ and interchanging the order of summations, we obtain

$$
\Phi(x, z)=V(x z) W(z),
$$

where $V(t)$ and $W(t)$ are the generating functions of the above sequences $v(k)$ and $w(k)$ :

$$
V(t)=\sum_{k=0}^{\infty} v(k) t^{k}, W(t)=\sum_{k=0}^{\infty} w(k) t^{k} .
$$

Noticing that $F(z)=V(z) W(z)$, we obtain

$$
J=\frac{N}{Z(M, N)} \oint_{\Gamma_{0}} \frac{[F(z)]^{N}}{z^{M}} \frac{V^{\prime}(z)}{V(z)} \frac{d z}{2 \pi i} .
$$

The generation functions of the sequences $w(k), v(k)$, and $f(k)$ from $10-12$ are

$$
V(t)=\frac{1-\nu t}{1-\mu t}, W(t)=\frac{1-\mu t}{1-t}, F(t)=\frac{1-\nu t}{1-t} .
$$

Then the above integrals can be evaluated in terms of hypergeometric functions. Specifically, the grand canonical partition function $[F(z)]^{N}$ and the function $[F(z)]^{N} V^{\prime}(z) / V(z)$, are generating functions of the ${ }_{2} F_{1}$ Gauss hypergeometric functions and $F_{1}$ Appell function respectively. The integrals extract from these series the coefficients of the terms of order $M$ and $(M-1)$, respectively (see Appendix B). Then, for the partition function we have

$$
Z(M, N)=\left(\begin{array}{c}
L-1 \\
M
\end{array}\right){ }_{2} F_{1}(-M,-N ; 1-L ; \nu),
$$

while the average number of particles jumping per unit time is

$$
J=\frac{(\mu-\nu) N M}{(L-1)} \frac{F_{1}(1-M ; 1-N, 1 ; 2-L ; \nu, \mu)}{{ }_{2} F_{1}(-M,-N ; 1-L ; \nu)} .
$$

When one of the arguments is zero, the $F_{1}$ Appell function is reduced to the ${ }_{2} F_{1}$ Gauss function (see appendix B). Therefore, in the limit $\mu=0$, i.e. the parallel update (PU) case, we recover the result obtained in [29]:

$$
J_{P U}=\frac{p}{1-p} \frac{N M}{(L-1)} \frac{{ }_{2} F_{1}\left(1-M ; 1-N, 2-L ;-\frac{p}{1-p}\right)}{{ }_{2} F_{1}\left(-M,-N ; 1-L ;-\frac{p}{1-p}\right)} .
$$

The $\nu=0$ case corresponds to the backward-sequential update (BSU), for which we have a formula

$$
J_{B S U}=\frac{p N M}{(L-1)}{ }_{2} F_{1}(1-M ; 1,2-L ; p),
$$

obtained in [39]. [There is a minor mistype in [39]: the factor $z$ corresponding to our $p$ is missing from the final expression, formula (16).] Also the $p=1$ limit of (18) was obtained in 24. It should be noted also that the $F_{1}$ Appell function is a two-variable reduction of the Lauricella hypergeometric function $F_{D}$, which depends on an arbitrary number of variables. The particle current in a particular example of ZRP, where, at most, one particle may jump from sites with $r \leq K$ particles with arbitrary probabilities $0<u(r)<1$ and from sites with $r>K$ with probability $u(r)=1$ was obtained in [40] in terms of Lauricella hypergeometric function $F_{D}$ of $K$ variables. Presumably, our case and the one studied in [40] can be unified within a larger class of processes.

The results we have just obtained give the exact formulas for the partition functions, from which we can also obtain the occupation number distribution, and the mean particle current on an arbitrary finite lattice. However, of physical interest is the thermodynamic limit,

$$
N \rightarrow \infty, M \rightarrow \infty, M / N=\rho=\text { const }
$$

It turns out that depending on the scale of the parameter $\lambda$, two different regimes naturally appear.

\section{B. Asymptotic analysis}

$$
\text { Saddle point method, } \lambda / N^{2} \rightarrow 0
$$

In the limit (19), we can try to evaluate the integrals (14) and (17) in the saddle point approximation. The integrals have the form

$$
\mathcal{I}_{N}(h(z), g(z))=\oint_{\Gamma_{0}} e^{N h(z)} g(z) \frac{d z}{2 \pi i z}
$$

where

$$
h(z)=\ln (1-\nu z)-\ln (1-z)-\rho \ln z .
$$


The critical points of the function $h(z)$ are defined by equation $h^{\prime}(z)=0$, which has two solutions

$$
z_{ \pm}=1+\frac{(1-\nu)}{2 c \nu}\left(1 \pm \sqrt{1+\frac{4(1-c) c \nu}{1-\nu}}\right)
$$

where $c=\rho /(1+\rho)$ is the concentration of particles in the ASEP-like system. The simple analysis shows that $0<z_{-}<1$ and $z_{+}>1, \Re h\left(z_{+}\right)<0$ and $\Re h\left(z_{-}\right)>0$.
Therefore

$$
z=z_{-}
$$

is the point which gives the dominant contribution to the integrals. We now choose the steepest descent contour being a circle of radius $z_{-}$with the center at the origin. Then the integral 20 asymptotically is

$$
\mathcal{I}_{N}(h(z), g(z))=\frac{e^{N h_{0}}}{\sqrt{2 \pi N\left|h_{2}\right|}}\left[g_{0}+\frac{1}{2 N}\left(\frac{g_{2}}{\left|h_{2}\right|}+\frac{g_{1} h_{3}}{h_{2}^{2}}+\frac{g_{0}}{4}\left(\frac{h_{4}}{h_{2}^{2}}+\frac{5 h_{3}^{2}}{3\left|h_{2}\right|^{3}}\right)\right)+O\left(N^{-2}\right)\right]
$$

where $g_{k}=\left.\left(\mathrm{i} z \partial_{z}\right)^{k} g(z)\right|_{z=z_{-}}$and $h_{k}=\left.\left(\mathrm{i} z \partial_{z}\right)^{k} h(z)\right|_{z=z_{-}}$. Choosing $g(z)=1$ we obtain the leading order of the partition function

$$
Z(M, N)=\mathcal{I}_{N}(h(z), 1) \simeq \frac{\exp \left(N h_{0}\right)}{\sqrt{2 \pi N\left|h_{2}\right|}}
$$

To obtain the occupation number distribution $P(n)$ using 15. we need also the value of $Z(M-n, N-1)$, which can be evaluated choosing $g(z)=z^{n} \exp [-h(z)]$. As a result, in the limit 19 for $n$ not too large $P(n) \simeq f(n) g\left(z_{-}\right)$, from where we have a usual Gibbs-like form

$$
\begin{aligned}
& P(n)=\lambda^{-1} \exp \left(-n / n^{*}-h\left(z_{-}\right)\right), n>0 \\
& P(0)=\exp \left(-h\left(z_{-}\right)\right),
\end{aligned}
$$

with $n^{*}=-1 / \ln z_{-}$, obtained before in [24]. This form of the distribution suggests that only sites with finite (mainly $n \lesssim n^{*}$ ) occupation numbers have a chance to appear in a typical configuration in the stationary state.

To interpret this results in terms of the gTASEP, we note that conditioned to occupied sites, $n>0$, the distribution obtained gives the cluster size distribution. It is the geometric distribution $P\left(l_{\mathrm{cl}}=n\right)=$ $\left(1-z_{-}\right) z_{-}^{n}$, with the mean cluster size equal to $\left\langle l_{\mathrm{cl}}\right\rangle=$ $z_{-}\left(1-z_{-}\right)^{-1}$. As $\lambda \rightarrow \infty$, the mean cluster size grows as $\left\langle l_{\mathrm{cl}}\right\rangle \sim \sqrt{\lambda / \rho}$, and, correspondingly, the mean number of clusters at the lattice is $M\left\langle l_{\mathrm{cl}}\right\rangle^{-1} \simeq L c^{3 / 2} / \sqrt{\lambda(1-c)}$. As discussed in the end of this section, the saddle point approximation is valid as far as $\lambda L^{-2} \rightarrow 0$. Therefore, these results hold as far as the number of clusters grows with the system size and their size is subextensive, i.e., much less than $L$.

To obtain the particle current we must also evaluate the ratio

$$
J=N \frac{\mathcal{I}_{N}(h(z), g(z))}{\mathcal{I}_{N}(h(z), 1)}
$$

with the function $g(z)=z V^{\prime}(z) / V(z)=(1-\mu z)^{-1}-$ $(1-\nu z)^{-1}$. The next to leading order finite size correction to the particle current has a universal meaning in context of KPZ theory, which will be discussed below. Therefore, we keep the terms of the asymptotic expansion up to the next to leading order, which yields

$$
J=N g_{0}+\frac{1}{2}\left(\frac{g_{1} h_{3}}{\left|h_{2}\right|^{2}}+\frac{g_{2}}{\left|h_{2}\right|}\right)+O\left(N^{-1}\right) .
$$

From the leading order we obtain the current-density relation (so-called flow diagram) for the gTASEP, which being expressed in terms of probabilities $p$ and $\mu$, reads as

$$
\begin{aligned}
j^{A S E P} & =\frac{c p(1+(1-2 c) \mu)}{2 \mu+2 c(p(1-\mu)-\mu)} \\
& -\frac{c p \sqrt{(1-\mu)(1-4(1-c) c(p-\mu)-\mu)}}{2 \mu+2 c(p(1-\mu)-\mu)}
\end{aligned}
$$

and reproduces the formula obtained in [24]. The particular cases of this expression are:

$\mu=0$, well known current-density relation for the TASEP with PU first obtained in studies of traffic models [4],

$$
j_{P U}=\frac{1}{2}(1-\sqrt{1-4 p c(1-c)})
$$

$\mu=p, \mathrm{BSU}$ 42,

$$
j_{B S U}=\frac{(1-c) c p}{1-c p}
$$

$\mu=1$, the limit of DA in which all particles finally stick together into a single giant cluster, which performs ordinary Bernoulli random walk,

$$
j_{D A}=c p
$$

In the case of PU, the current-density plot is symmetric due to particle-hole symmetry. The bigger the value of 
$\mu$, the more right skewed is the plot. In the DA limit, it degenerates into the linear function describing a random walk of a single particle making steps of length $M$.

The explicit expression of the next to leading order finite-size correction to the current given in $\mathrm{A} 4 \mathrm{P}$ of $\mathrm{Ap}$ pendix $\mathrm{A}$ is rather cumbersome. However, it is informative to look at it close to DA limit:

$$
\mu \rightarrow 1, \nu \rightarrow 1, p=\frac{\mu-\nu}{1-\nu}=\text { const. }
$$

It is convenient to describe this limit in terms of the parameter $\lambda$ defined in (3), for which the limit corresponds to $\lambda \rightarrow \infty$. Then the leading asymptotics in $\lambda$ is

$$
\begin{aligned}
j^{A S E P}(L) & -j^{A S E P}(\infty) \\
& =\frac{1}{L}\left[\frac{3 c p(1-p)}{4(1-c)}+O\left(\lambda^{-1 / 2}\right)\right]+O\left(L^{-2}\right) .
\end{aligned}
$$

Surprisingly the $1 / L$ correction saturates to the finite limit as $\lambda \rightarrow \infty$. However, the next orders' corrections diverge in this limit, so that the effective expansion parameter is $\sqrt{\lambda} / L$, from where we can estimate the range of validity of the expressions obtained. One can see that the correction becomes non-neglectable as soon as $\lambda$ is of the order of $L^{2}$. In fact, the very applicability of the saddle point method is violated at this scale. The reason for that is merging of the saddle points $z_{-}$and $z_{+}$with the pole of the function under the integral, which makes all the terms of the expansion of the function $h(z)$ effectively of the same order. Indeed, though we implicitly assumed that all the parameters of $h(z)$ are constants as $N$ grows, the saddle point method can still be applied with $N$-dependent parameters as far as the limit

$$
\lim _{N \rightarrow \infty}\left|\frac{N^{1-k / 2} h_{k}}{h_{2}^{k / 2}}\right|=0
$$

holds for $k \geq 3$, where $h_{k}$ is the $k$-th derivative of $h(z)$ evaluated at the saddle point $z_{-}$. In the limit $\lambda \rightarrow \infty$, the saddle points are as close to $z=1$ as $z_{ \pm}=1 \pm$ $\sqrt{1 / \rho \lambda}+O(1 / \lambda)$ and $h_{k}$ grows as $h_{k} \sim \lambda^{\frac{k-1}{2}}$. Therefore, the above limit holds as far as $\lambda N^{-2} \rightarrow 0$. The situation when $\lambda N^{-2} \rightarrow$ const $>0$, corresponding to the transition regime, requires a separate asymptotic analysis.

$$
\text { Transition regime, } \lambda N^{-2}=\text { const. }
$$

Let us consider the integral 14 representing the partition function $Z(M, N)$. To evaluate the integral asymptotically we note that the function under the integral has only two singularities in the complex plane $z=0$ and $z=1$, and, in particular, is analytic at infinity. Therefore we can deform the integration contour $\Gamma_{0}$ closed around the origin by a contour $\Gamma_{1}$ closed around $z=1$ :

$$
Z(M, N)=-\oint_{\Gamma_{1}} e^{N h(z)} \frac{d z}{2 \pi i z}
$$

Apart from specifying position of the contour with respect to singularities, we can choose it of any form. It is convenient to integrate over a small circle centered at $z=1$ going close to the saddle points. Then, instead of the the function $h(z)$, one can use its asymptotic expansion at this contour. Choosing

$$
z=1+\frac{e^{i \varphi}}{\sqrt{\rho \lambda}}
$$

we get

$$
h(z)=-2 \sqrt{\frac{\rho}{\lambda}} \cos \varphi+O(1 / \lambda)
$$

and for the integral we obtain

$$
\begin{aligned}
Z(M, N) & =\frac{-1}{\sqrt{\rho \lambda}} \int_{0}^{2 \pi} e^{-2 N \sqrt{\rho / \lambda} \cos \varphi+\mathrm{i} \varphi} \frac{d \varphi}{2 \pi}+O\left(N^{-2}\right) \\
& \simeq \frac{\theta}{2 M} I_{1}(\theta)
\end{aligned}
$$

where $I_{k}(y)$ is the modified Bessel function of the first kind (for the definition and properties see appendix C) and we introduced the scaling parameter

$$
\theta=2 N \sqrt{\frac{\rho}{\lambda}},
$$

which is finite in the limit under consideration and is supposed to control the transition from the KPZ to the DA regime. For the occupation number probability distribution $P(n)$ we also need $Z(M-n, N-1)$, which in the leading order is obtained from the above equation by replacing: $M \rightarrow M-n, \rho \rightarrow \rho-n / N$ and $\theta \rightarrow \theta \sqrt{1-n / M}$. Then for the occupation number probability distribution we have

$$
\begin{aligned}
P(0) & \simeq 1-\frac{\theta}{2 N} \frac{I_{0}(\theta)}{I_{1}(\theta)} \\
P(n) & \simeq \frac{\theta^{2}}{4 N M} \frac{I_{1}\left(\theta \sqrt{1-\frac{n}{M}}\right)}{I_{1}(\theta) \sqrt{1-\frac{n}{M}}}, \quad 0<n<M, \\
P(M) & \simeq \frac{\theta}{2 N} \frac{1}{I_{1}(\theta)} .
\end{aligned}
$$

Here we kept only the leading order of the expansion of $P(n)$ for $n>0$ and two highest orders in $P(0)$ (the latter can actually be obtained from the former by normalization). It is clear from the first line that the typical configuration contains only a finite number of sites are occupied. Consider now the distribution of the random variable $\chi=n / M \in[0,1]$ conditioned at $n>0$, i.e. only the occupied sites are counted. To evaluate the conditional probability, we divide the right-hand side of 30 and (31) by $\operatorname{Prob}(n>0)=(1-P(0))$. The distribution obtained has well defined limiting behavior as $N \rightarrow \infty$ being parametrized by single parameter $\theta$ :

$$
\begin{aligned}
& \operatorname{Prob}(\chi=1)=\frac{1}{I_{0}(\theta)} \\
& \operatorname{Prob}(\chi<x)=\frac{\theta}{2 I_{0}(\theta)} \int_{0}^{x} \frac{I_{1}(\theta \sqrt{1-y})}{\sqrt{1-y}} d y .
\end{aligned}
$$


A finite fraction of the distribution is concentrated at single point $\chi=1$ and the rest is the continuous distribution on $[0,1)$. In terms of the gTASEP the probability $(32)$ is exactly the limiting fraction of time, which all particles spend in a single cluster. The rest of the time finitely many clusters of macroscopic size, $n \sim M$, exist on a lattice. For $\chi<1$ the distribution of the fraction of all particles contained in a given cluster converges to the continuous distribution (33). The mean size of a cluster is $\left\langle l_{\mathrm{cl}}\right\rangle \simeq 2 M I_{1}(\theta)\left[\theta I_{0}(\theta)\right]^{-1}$ and, $M /\left\langle l_{\mathrm{cl}}\right\rangle$ is the expected number of clusters, which starting from one at $\theta=0$ approaches a linear growth $M /\left\langle l_{\mathrm{cl}}\right\rangle \simeq \theta / 2$ as $\theta \rightarrow \infty$.

\section{Transfer matrix approach and correlation length}

In addition to the above calculations with the ZRP-like system, which is suitable for characterizing the current and cluster size distribution, one can look at the system directly in the ASEP formulation, which is more appropriate for for study of the stationary correlation functions. The stationary measure of particle configurations in the TASEP-like system is similar to that of the ZRPlike system up to the symmetry with respect to lattice rotation. Specifically, we must replace every site occupied with $n$ particles with a cluster of $n$ particles plus one empty site. Looking at formulas for the stationary measure of a ZRP-like system (8) and (12) we assign the weight $(1-\nu)$ to each cluster and the weight 1 to each empty site. It is convenient to study the system in the grand canonical ensemble, where in addition to the above cluster weights we attach the fugacity $z$ to each particle. Then, the stationary probability of a particle configuration $\boldsymbol{\tau}=\left(\tau_{1}, \ldots, \tau_{L}\right)$, with occupation numbers $\tau_{1}, \ldots, \tau_{L}=0,1$, will be

$$
P_{s t}(\boldsymbol{\tau})=\frac{1}{\mathcal{Z}_{L}(z)} T_{\tau_{1}, \tau_{2}} \ldots T_{\tau_{L-1}, \tau_{L}} T_{\tau_{L}, \tau_{1}},
$$

where $T_{0,0}=1, T_{0,1}=T_{1,0}=\sqrt{z(1-\nu)}$, and $T_{1,1}=z$. This measure is similar to the Gibbs measure of the 1D Ising model, as was first observed in [41] in the context of the TASEP with PU. Correspondingly, for periodic boundary conditions the partition function is given by the trace of $L$-th power of the transfer matrix

$$
\mathcal{Z}_{L}(z)=\operatorname{Tr} T^{L}=\lambda_{1}^{L}+\lambda_{2}^{L},
$$

where

$$
\begin{aligned}
& \lambda_{1}=\frac{1}{2}\left(1+z+\sqrt{(z+1)^{2}-4 \nu z}\right), \\
& \lambda_{2}=\frac{1}{2}\left(1+z-\sqrt{(z+1)^{2}-4 \nu z}\right),
\end{aligned}
$$

are eigenvalues of the matrix $T$, defined so that $\lambda_{1}>$ $\lambda_{2} \geq 0$. The largest eigenvalue $\lambda_{1}$ defines the specific free energy,

$$
f(z)=-\lim _{L \rightarrow \infty} \frac{\ln \mathcal{Z}_{L}(z)}{L}=-\ln \lambda_{1} .
$$

The density of particles is fixed by the thermodynamic relation

$$
c=-z \partial_{z} f(z) .
$$

This is the quadratic equation for $z$ with two roots, which interchange under replacement $c \longleftrightarrow 1-c$. Which one to choose is to be decided from direct evaluation of the particle density, i.e. of one-point correlation function. In general to evaluate $s$-point correlation functions of the form $\left\langle\tau_{k_{1}} \ldots \tau_{k_{s}}\right\rangle$, where $\langle a\rangle$ is the notation for expectation value of the random variable $a$, one has to insert the matrix

$$
\widehat{\tau}=\left(\begin{array}{ll}
0 & 0 \\
0 & 1
\end{array}\right)
$$

into the product of transfer matrices in the places corresponding to sites $k_{1}, \ldots, k_{s}$ :

$$
\left\langle\tau_{k_{1}} \ldots \tau_{k_{s}}\right\rangle=\frac{\operatorname{Tr}\left[T^{k_{1}} \widehat{\tau} T^{k_{1}+k_{2}} \widehat{\tau} \ldots \widehat{\tau} T^{L-\left(k_{1}+\cdots+k_{s}\right)}\right]}{\mathcal{Z}_{L}(z)} .
$$

To evaluate expressions of this kind we also need the eigenvectors of $T$,

$$
\mathbf{v}_{1}=\left[\begin{array}{c}
\left(\frac{\sqrt{(z+1)^{2}-4 \nu z}-z+1}{2 \sqrt{(z+1)^{2}-4 \nu z}}\right)^{\frac{1}{2}} \\
\left(\frac{\sqrt{(z+1)^{2}-4 \nu z}+z-1}{2 \sqrt{(z+1)^{2}-4 \nu z}}\right)^{\frac{1}{2}}
\end{array}\right],
$$

and

$$
\mathbf{v}_{2}=\left[\begin{array}{c}
-\left(\frac{\sqrt{(z+1)^{2}-4 \nu z}+z-1}{2 \sqrt{(z+1)^{2}-4 \nu z}}\right)^{\frac{1}{2}} \\
\left(\frac{\sqrt{(z+1)^{2}-4 \nu z}-z+1}{2 \sqrt{(z+1)^{2}-4 \nu z}}\right)^{\frac{1}{2}}
\end{array}\right],
$$

corresponding to $\lambda_{1}$ and $\lambda_{2}$ respectively, which are normalized to $\left\|v_{1}\right\|=\left\|v_{2}\right\|=1$. Verifying the identity $c=\langle\tau\rangle=\operatorname{Tr}\left(\widehat{\tau} T^{L}\right) \simeq\left(\mathbf{v}_{1}, \widehat{\tau} \mathbf{v}_{1}\right)$, we see that the root of (34) to be chosen is

$$
z^{*}=1-2\left(1+\frac{\sqrt{(1-\nu)\left(1-\nu(1-2 c)^{2}\right)}}{(1-2 c)(1-\nu)}\right)^{-1}
$$

With the use of relations $\left(\mathbf{v}_{1}, \widehat{\tau} \mathbf{v}_{1}\right)=c,\left(\mathbf{v}_{2}, \widehat{\tau} \mathbf{v}_{2}\right)=(1-$ $c)$, and $\left(\mathbf{v}_{1}, \widehat{\tau} \mathbf{v}_{2}\right)=\sqrt{c(1-c)}$ the two point correlation function is given by 


$$
\left\langle\tau_{1} \tau_{1+k}\right\rangle=\frac{c^{2}+(1-c)^{2} e^{-L / \xi}+c(1-c)\left(e^{-k / \xi}+e^{-(L-k) / \xi}\right)}{1+e^{-L / \xi}}
$$

where

$$
\xi \equiv \frac{1}{\ln \left(\lambda_{1} / \lambda_{2}\right)}=-\left[\ln \left(1-\frac{2}{1+\sqrt{\frac{1-(1-2 c)^{2} \nu}{1-\nu}}}\right)\right]^{-1}
$$

is the correlation length. When the correlation length is finite, i.e. for $\nu<1$, the two-point correlator becomes a product of one point correlators $\left\langle\tau_{1} \tau_{1+k}\right\rangle \rightarrow c^{2}$ at large distances, $L \gg k \rightarrow \infty$. As expected, the covariance decays exponentially in the range $\xi \ll k \ll L$,

$$
C(k) \equiv\left\langle\tau_{1} \tau_{1+k}\right\rangle-\left\langle\tau_{1}\right\rangle\left\langle\tau_{1+k}\right\rangle \simeq c(1-c) e^{-k / \xi},
$$

which justifies $\xi$ being the correlation length. As $\nu \rightarrow 1$, i.e., $\lambda \rightarrow \infty$, the correlation length diverges as

$$
\xi \simeq \sqrt{\lambda c(1-c)} .
$$

In the transition regime, $\lambda \sim L^{2}$, the correlation range is of the order of the system size $L$. Expressed in terms of the distance $r$ measured in units of the system size the covariance has the form

$$
C(L r)=\frac{(1-2 c) e^{-1 / \widetilde{\xi}}+c(1-c)\left(e^{-r / \widetilde{\xi}}+e^{-(1-r) / \widetilde{\xi}}\right)}{1+e^{-1 / \widetilde{\xi}}},
$$

where $\widetilde{\xi}=2 c(1-c) / \theta$ is the effective correlation length in the system size scale, which depends on the transition parameter $\theta$ defined in (28).

\section{STATISTICS OF PARTICLE CURRENT}

\section{A. Bethe ansatz and method by Derrida-Lebowitz}

To characterize current fluctuations we introduce a deformed Markov matrix $\mathbf{M}^{\gamma}$, depending on an auxiliary parameter $\gamma$, where every particle step is supplied with an extra weight $\exp \gamma$. Then the matrix elements are defined as follows

$$
\mathbf{M}_{\mathbf{n}, \mathbf{n}^{\prime}}^{\gamma}=\mathbf{M}_{\mathbf{n}, \mathbf{n}^{\prime}} \exp \left(\gamma \mathcal{N}\left(\mathbf{n}, \mathbf{n}^{\prime}\right)\right)
$$

where $\mathbf{M}_{\mathbf{n}, \mathbf{n}^{\prime}}$ - are matrix elements of the original Markov matrix and $\mathcal{N}\left(\mathbf{n}, \mathbf{n}^{\prime}\right)$ is the number of particle jumps in the one-step transition from $\mathbf{n}^{\prime}$ to $\mathbf{n}$. This matrix governs the evolution of the generating function $G_{t}(\mathbf{n}, \gamma)=\sum_{Y=0}^{\infty} e^{\gamma Y} P_{t}(\mathbf{n}, Y)$, of the joint probability $P_{t}(\mathbf{n}, Y)$ for the system to be in configuration $\mathbf{n}$ at time $t$, while the total distance $Y_{t}$ traveled by all particles is equal to $Y$,

$$
\mathbf{G}_{t+1}=\mathbf{M}^{\gamma} \mathbf{G}_{t}
$$

where $\mathbf{G}_{t}$ is the column vector with components $G_{t}(\mathbf{n}, \gamma)$. The generating function $\left\langle e^{\gamma Y_{t}}\right\rangle$ of moments of $Y_{t}$ is a sum of $\sum_{\mathbf{n}} G_{t}(\mathbf{n}, \gamma)$ over all configurations. Its large time behavior is dominated by the eigenvalue $\Lambda_{0}(\gamma)$ of the matrix $\mathbf{M}^{\gamma}$ with the largest real part, and, hence, the logarithm of $\Lambda_{0}(\gamma)$ is the scaled generating function of cumulants of $Y_{t}$,

$$
\ln \Lambda_{0}(\gamma)=\lim _{t \rightarrow \infty} \frac{\ln \left\langle e^{\gamma Y_{t}}\right\rangle}{t} .
$$

The diagonalization of matrix $\mathbf{M}^{\gamma}$ using the Bethe ansatz technique was described in details in [25, 26] for both ASEP and ZRP-like versions of our system. Let us first briefly discuss the latter. Alternatively to the set of occupation numbers $\mathbf{n}$, it is convenient to describe particle configurations in terms of coordinates of particles on the lattice $\mathcal{L}$,

$$
\mathbf{y}=\left(1 \leq y_{1} \leq \cdots \leq y_{M} \leq N\right)
$$

in the same way as we do for the ASEP, except that the coordinates are weakly ordered, because many particles in a site are allowed. Then, the components of eigenvectors $\boldsymbol{\Psi}$ of $\mathbf{M}^{\gamma}$ are looked for in the form

$$
\Psi_{\mathbf{n}}(\mathbf{z})=P_{s t}(\mathbf{n}) \Psi^{0}(\mathbf{y} \mid \mathbf{z}),
$$

where $P_{s t}(\mathbf{n})$ is the stationary state weight of particle configuration, and

$$
\Psi^{0}(\mathbf{y} \mid \mathbf{z})=\sum_{\sigma \in S_{M}} A_{\sigma} z_{\sigma_{1}}^{y_{1}} \ldots z_{\sigma_{M}}^{y_{M}}
$$

is the Bethe function depending on $M$-tuple $\mathbf{z}=$ $\left(z_{1}, \ldots, z_{M}\right)$ of complex numbers to be defined later. Here, $\mathbf{y}$ are weakly increasing coordinates of particles corresponding to the occupation numbers $\mathbf{n}$, the summation is over the permutations $\sigma=\left(\sigma_{1}, \ldots, \sigma_{M}\right)$ of the numbers $(1, \ldots, M)$, and $A_{\sigma}$ are the permutation-dependent coefficients defined by relation

$$
\frac{A \ldots i j \ldots}{A \ldots j i \ldots}=-\frac{\left(1-e^{-\gamma} z_{i}\right)\left(\nu-e^{-\gamma} z_{j}\right)}{\left(1-e^{-\gamma} z_{j}\right)\left(\nu-e^{-\gamma} z_{i}\right)} .
$$

One can show that action of the matrix $\mathbf{M}^{\gamma}$ on $\boldsymbol{\Psi}$ is reduced to multiplication by the eigenvalue

$$
\Lambda(\gamma)=\prod_{i=1}^{M}\left(e^{\gamma} p z_{i}^{-1}+(1-p)\right),
$$

provided that the Bethe function satisfies periodic boundary conditions $\Psi^{0}\left(y_{1}, \ldots, y_{M} \mid \mathbf{z}\right)=$ 
$\Psi^{0}\left(y_{2}, \ldots, y_{M}, y_{1}+N \mid \mathbf{z}\right)$, which are equivalent to the system of $M$ algebraic Bethe ansatz equations (BAEs),

$$
z_{i}^{N}=(-1)^{M-1} \prod_{j=1}^{M} \frac{\left(1-e^{-\gamma} z_{i}\right)\left(\nu-e^{-\gamma} z_{j}\right)}{\left(1-e^{-\gamma} z_{j}\right)\left(\nu-e^{-\gamma} z_{i}\right)} .
$$

for the numbers $z_{1}, \ldots, z_{M}$.

Though the above analysis is applied to the ZRP-like system, the minor modification is required to the ASEP. The eigenvector of the corresponding Markov matrix is that in 40 , except that the particle coordinates are read off in a different way. Specifically, the ASEP coordinates $\mathbf{x}$ are obtained from the ZRP coordinates by a shift

$$
\left(x_{1}, x_{2}, \ldots, x_{M}\right)=\left(y_{1}, y_{2}+1 \ldots, y_{M}+M-1\right),
$$

which ensures them to be strictly increasing as necessary. One can also look for the eigenvector right in the form 40 in terms of the ASEP coordinates $\mathbf{x}$. Then we will have to multiply the ratio of amplitudes (41) by the factor $z_{i} / z_{j}$. The form 42 of the eigenvalues stays the same and the periodic boundary conditions $\Psi^{0}\left(x_{1}, \ldots, x_{N} \mid \mathbf{z}\right)=\Psi^{0}\left(x_{2}, \ldots, x_{N}, x_{1}+L \mid \mathbf{z}\right)$ yield the $\mathrm{BAE}$

$$
z_{i}^{L}=(-1)^{M-1} \prod_{j=1}^{M} \frac{z_{i}\left(1-e^{-\gamma} z_{i}\right)\left(\nu-e^{-\gamma} z_{j}\right)}{z_{j}\left(1-e^{-\gamma} z_{j}\right)\left(\nu-e^{-\gamma} z_{i}\right)}
$$

which are different from $\sqrt{43}$ in a single factor $\prod_{j=1}^{M} z_{j}$.

The problem of finding the largest eigenvalue for both models is reduced to identifying a particular solution of the BAE corresponding to the ground state. To this end, we note that in the limit $\gamma \rightarrow 0$ the matrix $\mathbf{M}^{\gamma}$ turns to the transition probability matrix $\mathbf{M}$ having the largest eigenvalue equal to one, so that we expect $\Lambda_{0}(\gamma) \rightarrow 1$ as $\gamma \rightarrow 0$. In addition, the corresponding eigenvector becomes the stationary state in this limit, which can be obtained from (39) and (40) by setting $z_{1}=\cdots=z_{M}=1$. Taking the product of all $M$ equations in both systems, we see that all solutions satisfy the constraints $\left(\prod_{j=1}^{M} z_{j}\right)^{N}=1$ and $\left(\prod_{j=1}^{M} z_{j}\right)^{L}=1$ for 43 and 44 , respectively. Therefore, the sets of solutions of BAE can be classified into sectors, where the product of Bethe roots equals different roots of unity independent of $\gamma$. In particular, continuing the $\gamma=0$ limit to arbitrary values of $\gamma$, we see that in both systems the ground states belong to the sector

$$
\prod_{j=1}^{M} z_{j}=1
$$

where the systems (43) and (44) are identical and we can use either of them to obtain the eigenvalue $\Lambda_{0}(\gamma)$. The product of the Bethe roots is the factor that the Bethe eigenfunction multiplies by under the unit translation, i.e. the eigenvalue of the translation operator commuting with the matrix $\mathbf{M}^{\gamma}$. Its value reflects the translation invariance of the ground state mentioned before.

To find the solution of 43 , we first make a change of variables,

$$
z_{i}=e^{\gamma} \frac{1-\nu u_{i}}{1-u_{i}}
$$

In the variables $u_{i}$, the BAE and the eigenvalue $\Lambda(\gamma)$ simplify to the following form

$$
\begin{aligned}
\left(\frac{1-\nu u_{i}}{1-u_{i}}\right)^{N} e^{N \gamma} & =(-1)^{M-1} \prod_{j=1}^{M} \frac{u_{i}}{u_{j}} \\
\Lambda(\gamma) & =\prod_{i=1}^{M}\left(\frac{1-\mu u_{i}}{1-\nu u_{i}}\right) .
\end{aligned}
$$

The method used by Derrida and Lebowitz to find eigenvalues for the TASEP is based on the observation that the solutions of BAE can be found among the roots of a single polynomial,

$$
\mathcal{P}(u)=(1-\nu u)^{N} B-(1-u)^{N} u^{M},
$$

where $B=(-1)^{M-1} e^{\gamma N} \prod_{j=1}^{M} u_{j}$ is the parameter that itself is a function of the solution. What we actually need is to evaluate the sums of values that particular functions take on the roots from the solution of interest. These sums can be evaluated using the Cauchy theorem,

$$
\sum_{i=1}^{M} f\left(u_{j}\right)=\oint_{\Gamma_{0}} f(u) \frac{\mathcal{P}^{\prime}(u)}{\mathcal{P}(u)} \frac{d u}{2 \pi \mathrm{i}}
$$

where the integration is over the contour enclosing all the necessary roots and the function $f(u)$ is analytic inside the contour. In our case the roots from the solution corresponding to the ground state are those $M$ roots $z_{1}, \ldots, z_{M}$, which approach one as $\gamma \rightarrow 0$ or zero in terms of the variables $u_{1}, \ldots, u_{M}$. Choosing the function $f(u)$ in the form $f(u)=\ln [(1-\mu u) /(1-\nu u)]$ we obtain after the integration by parts the logarithm of the largest eigenvalue as function of $B$ :

$$
\ln \Lambda_{0}(\gamma)=(\mu-\nu) \oint_{\Gamma_{0}} \frac{\ln \left[1-\frac{B(1-\nu u)^{N}}{(1-u)^{N} u^{M}}\right]}{(1-\mu u)(1-\nu u)} \frac{d u}{2 \pi i}
$$

Here the integration is over the contour satisfying to the condition $\left|B(1-\nu u)^{N} /(1-u)^{N} u^{M}\right|<1$ and enclosing $M$ roots of $\mathcal{P}(u)$ located near the origin. Note that the contour does not cross any branch cuts of the logarithm, which can be chosen connecting $M$ roots inside the contour to the origin and the other $N$ roots outside the contour to $u=1$. Such a contour exists if $|B|$ is small enough. The relation of $\gamma$ and $B$ can be recovered from 
the translation invariance condition (45), which after taking a logarithm and going to the variables $u_{i}$ yields

$$
\gamma=\frac{1-\nu}{M} \oint_{\Gamma_{0}} \frac{\ln \left(1-\frac{B(1-\nu u)^{N}}{(1-u)^{N} u^{M}}\right)}{(1-u)(1-\nu u)} \frac{d u}{2 \pi i}
$$

Note also, that what we actually integrated by parts to arrive at formulas (50) and (51) were not exactly the orig- inal expressions given by 49 , but the ones obtained by addition of terms analytic inside the contour of integration, which, hence, are integrated out to zero.

To evaluate the integrals we use a series expansion of the logarithms in powers of $B$ and integrate the resulting series term by term. Integrations can explicitly be performed in terms of the Appell and Gauss hypergeometric functions:

$$
\begin{aligned}
\ln \Lambda_{0}(\gamma) & =-(\mu-\nu) \sum_{n=1}^{\infty} \frac{B^{n}}{n}\left(\begin{array}{c}
L n-2 \\
M n-1
\end{array}\right) F_{1}(1-n M ; 1-n N, 1 ; 2-n L ; \nu, \mu) \\
\gamma & =-\frac{1-\nu}{M} \sum_{n=1}^{\infty} \frac{B^{n}}{n}\left(\begin{array}{c}
L n-1 \\
M n-1
\end{array}\right){ }_{2} F_{1}(1-M n, 1-N n ; 1-n L ; \nu)
\end{aligned}
$$

The scaled cumulants

$$
\left.c_{n} \equiv \lim _{t \rightarrow \infty} \frac{\partial^{n}}{t \partial \gamma^{n}}\left\langle e^{\gamma Y_{t}}\right\rangle\right|_{\gamma=0}=\left.\frac{\partial^{n}}{\partial \gamma^{n}} \ln \Lambda_{0}(\gamma)\right|_{\gamma=0} .
$$

of the particle current $Y_{t}$ can be obtained as the coefficients of the power expansion of $\ln \Lambda_{0}(\gamma)$ in $\gamma$, which can be constructed to any finite order by eliminating $B$ between the two series. In particular, using the Eu- ler transformation for hypergeometric functions $\mathrm{B} 1$ the first scaled cumulant $c_{1}=\lim _{t \rightarrow \infty} t^{-1}\left\langle Y_{t}\right\rangle$, the number of particle jumps per unit time, can be shown to coincide with $J$ from (18) obtained by averaging over the stationary state. The second cumulant, the scaled variance of $Y_{t}$, is related to the diffusion coefficient for a particle $\Delta=M^{-2} c_{2}$. The exact value of the latter is

$$
\begin{array}{r}
\Delta=\lambda p \frac{\left(\begin{array}{c}
2 L-2 \\
2 M-1
\end{array}\right)}{\left(\begin{array}{c}
L-1 \\
M-1
\end{array}\right)}\left[\frac{(2 L-1)}{2(L-1)} \frac{F_{1}(1-M ; 1-N, 1 ; 2-L ; \nu, \mu){ }_{2} F_{1}(1-2 M, 1-2 N, 1-2 L ; \nu)}{\left[{ }_{2} F_{1}(1-M, 1-N, 1-L ; \nu)\right]^{3}}\right. \\
\left.-\frac{F_{1}(1-2 M ; 1-2 N, 1 ; 2-2 L ; \nu, \mu)}{\left[{ }_{2} F_{1}(1-M, 1-N, 1-L ; \nu)\right]^{2}}\right]
\end{array}
$$

from which, using the identities for Gauss and Appell functions we can recover the corresponding quantities for particular cases of PU, $\mu=0, \mathrm{BSU}, \nu=0$ and the DA limit $\mu \rightarrow \nu=1, p=$ const. The exact formula of cumulant $c_{n}$ is already rather cumbersome for $n=2$, and it becomes more and more complicated as $n$ grows. Of major interest is the scaling behavior of the cumulants and the whole function $\Lambda_{0}(\gamma)$, which is also related to the LDF of particle current.

\section{B. Scaling limits}

We would like to investigate the thermodynamic limit

$$
M, N \rightarrow \infty, M / N=\rho .
$$

The structure of terms of the series obtained for $\Lambda_{0}(\gamma)$ and $\gamma$ is very similar to that of the integrals analyzed above for the partition function and average current. Thus, the same asymptotic analysis is applicable. Again, depending on the scale of $\lambda$ there are two different regimes: the first, where the integrals can be analyzed in the saddle point approximation and the second where the integrals can be evaluated in terms of modified Bessel functions. It is worth emphasizing again that the saddle point approximation is valid at any scale of $\lambda$ satisfying $\lambda N^{-2} \rightarrow 0$. Thus, the universal KPZ scaling function obtained in this approximation holds through a range of scales, with the scale entering only to the non-universal scaling constants. Then, the as the parameter $\lambda N^{-2}$ varies from zero to infinity, the KPZ-Gauss transition 
takes place.

$$
\text { 1. KPZ regime, } \lambda N^{-2} \rightarrow 0, \gamma \rightarrow 0, \gamma \lambda^{1 / 4} N^{3 / 2}=\text { const }
$$

Up to the $1 / n$ factor and the common factors before the integrals the terms of the order $n$ of series $(52)$ and $(53)$ are given by the integrals $\mathcal{I}_{n N}(h(z), g(z))$ of the form 20 with the function $h(z)$ defined in (21) and instead of the function $g(z)$ we substitute $r(z)=z[(1-\mu z)(1-\nu z)]^{-1}$ for $\Lambda_{0}(\gamma)$ series and $s(z)=z[(1-z)(1-\nu z)]^{-1}$ for the terms of $\gamma$ series. To obtain the meaningful precision it is enough to keep only leading order terms in asymptotics of $\gamma$ and the next to the leading order terms for the eigenvalue. Evaluating $\mathcal{I}_{n N}(h(z), r(z))$ and $\mathcal{I}_{n N}(h(z), s(z))$ with the help of (23) in two leading orders we obtain the universal scaling form obtained first in 8,

$$
\ln \Lambda(\gamma)=J_{\infty} \gamma+a N^{-3 / 2} G\left(b N^{3 / 2} \gamma\right),
$$

where $G(z)$ has a parametric representation

$$
G(z)=-\mathrm{Li}_{5 / 2}(t), \quad z=-\mathrm{Li}_{3 / 2}(t),
$$

via the polylogarithm function $\operatorname{Li}_{s}(x)=\sum_{i>0} x^{i} / i^{s}$. The infinite volume current

$$
J_{\infty}=M p r_{0} / s_{0}=L j^{A S E P}
$$

coincides with the particle current obtained from the averaging over stationary state, the coefficients $a$ and $b$,

$$
a=\frac{\mu-\nu}{2 \sqrt{2 \pi\left|h_{2}\right|}}\left(\frac{r_{2}-s_{2} / s_{0}}{\left|h_{2}\right|}+\frac{\left(r_{1}-s_{1} / s_{0}\right) h_{3}}{h_{2}^{2}}\right)
$$

and

$$
b=\frac{\sqrt{2 \pi\left|h_{2}\right|}}{\rho s_{0}(1-\nu)}
$$

are the non-universal model-dependent constants expressed via the coefficients of expansion of the functions $h(z), r(z), s(z)$ in the dominant saddle point $z_{-}$: $r_{k}=\left.\left(\mathrm{i} z \partial_{z}\right)^{k} r(z)\right|_{z=z_{-}}, s_{k}=\left.\left(\mathrm{i} z \partial_{z}\right)^{k} s(z)\right|_{z=z_{-}}$and $h_{k}=$ $\left.\left(\mathrm{i} z \partial_{z}\right)^{k} h(z)\right|_{z=z_{-}}$. The explicit expressions of these constants can be found in Appendix A.

It is clear from (54) that nontrivial scaling occurs, when $\gamma$ is of order of $b^{-1} N^{-3 / 2}$, which is of order of $\lambda^{-1 / 4} N^{-3 / 2}$ as $\lambda \rightarrow \infty$. The scaling form (54) suggests that at large time deviations of the time-averaged current from its thermodynamic value are of the form

$$
\operatorname{Prob}\left(Y_{t} / t<y\right) \sim \exp \left[-a t N^{-3 / 2} \widehat{G}\left(\frac{y-J_{\infty}}{a b}\right)\right],
$$

where the scaling function $\widehat{G}(x)=\sup _{t}(x t-G(t))$ is a Legendre transform of function $G(t)$. Appearance of the factor $t N^{-3 / 2}$ is the universal KPZ-specific feature, which is akin to the fact that the dynamical exponent of the KPZ class is $z=3 / 2$. Specifically, the term at $N^{-3 / 2}$ is the parameter, which is supposed to be large, for the large deviation approximation to be good. This is in correspondence with the results of [8], up the fact that factor $t N^{-3 / 2}$ is corrected by the prefactor $a$, which decays as $a \sim \lambda^{-1 / 4}$ as $\lambda \rightarrow \infty$. When $\lambda$ grows with $N$ as $\lambda \sim N^{\alpha}$, we expect that the dynamical exponent varies continuously as $z=3 / 2+\alpha / 4$ from KPZ, $z=3 / 2$, to diffusive, $z=2$, value as $\alpha$ varies from $\alpha=0$ to $\alpha=2$. As was discussed above, the method of asymptotical analysis used is valid also for $\lambda$ growing with $N$ slower than $N^{2}$. Hence, the applicability of the DerridaLebowitz scaling form of the LDF extends to systems with larger than KPZ characteristic time scales, until the scaling becomes diffusive, $t \sim N^{2}$. In the DA limit all particles stick together into the cluster, which performs an ordinary random walk making $M$-step jumps at a time. The central part of its LDF is expected to be pure Gaussian $\operatorname{Prob}\left(Y_{t} / t<y\right) \sim \exp \left(-t M^{-2} y^{2} / 2\right)$. This indicates that the transition regime is expected on the scale $\lambda \sim N^{2}$.

The cumulants of $Y_{t}$ can be obtained by differentiating the function (54). In particular, from the first derivative we obtain the mean number of particle jumps per unit time up to the first order finite size correction that was already obtained in (26). Note that it is the value of this correction,

$$
\left(j^{A S E P}(L)-j^{A S E P}((\infty)) L=a b,\right.
$$

which is the denominator of the argument of the LDF $\hat{G}$ in 56. As noted in [8] the applicability of the scaling form of the LDF obtained is limited by the condition that the argument of the function $\widehat{G}(x)$ is of the order of one. Therefore, the denominator plays the role of the scale for the deviation of time-averaged number of jumps from its mean value, in which the scaling form (56) is valid. Remarkably, its value stays finite when $\lambda$ grows to infinity. The diffusion coefficient for one particle, related to the second cumulant, is

$$
\Delta=\frac{(1-c)^{3 / 2}}{c^{2}} \frac{b^{2} a}{2 \sqrt{2 L}},
$$

decaying as $L^{-1 / 2}$, which is specific for the KPZ class. In the limit $\lambda \rightarrow \infty$, we have

$$
\Delta \simeq \frac{\lambda^{1 / 4}}{\sqrt{L}} \frac{3}{4} \sqrt{\frac{\pi}{2}} \frac{p(1-p)}{[c(1-c)]^{1 / 4}}
$$

which again signals that when $\lambda \sim L^{2}$ the motion of particles changes from subdiffusive to diffusive. However, as it was discussed above, the saddle point method fails at this scale of $\lambda$ and we should again use different asymptotic analysis. 


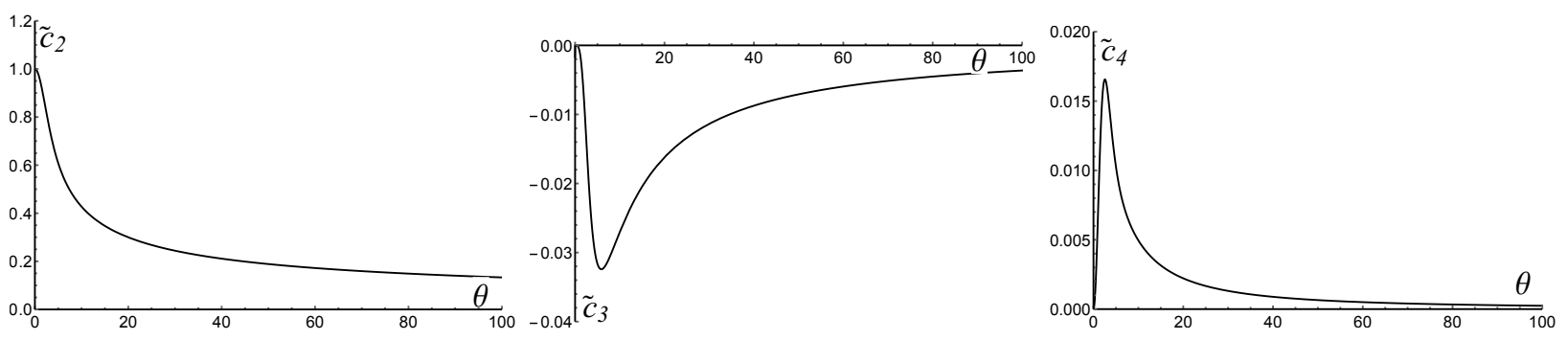

Figure 2: The rescaled cumulants of total number of particle jumps $\tilde{c}_{n}$ vs $\theta$ in the transition regime $N \rightarrow \infty, \lambda N^{-2}=$ const

2. The transition regime: $\lambda / N^{2}=$ const, $\gamma N^{2}=$ const

When $\lambda \sim N^{2}$, the integrals $\mathcal{I}_{n N}(h(z), r(z))$ and $\mathcal{I}_{n N}(h(z), s(z))$ can be evaluated in terms of the modified Bessel functions of the first kind as in the second part of Sec. IIIB As a result we obtain the parametrically defined function $\ln \Lambda(\gamma)$

$$
\ln \Lambda(\gamma)=\gamma p M+N^{-2} p(1-p) \mathcal{G}_{\theta}\left(N^{2} \rho \gamma\right),
$$

where the function $\mathcal{G}_{\theta}(t)$ depending on the transition parameter $\theta$ defined in 28 has the following parametric form:

$$
\mathcal{G}_{\theta}(t)=\frac{\theta^{2}}{4} \sum_{k=1}^{\infty} I_{2}(k \theta) \frac{B^{k}}{k}, \quad t=-\frac{\theta}{2} \sum_{k=1}^{\infty} I_{1}(k \theta) \frac{B^{k}}{k} .
$$

To observe the nontrivial scaling, $\gamma$ must scale with $N$ so that the limit $\gamma N^{2}=$ const holds.

Using an asymptotic form of the modified Bessel functions (C1) for large $\theta$ and small $t$, such that $t^{2} \theta=$ const, we find

$$
\mathcal{G}_{\theta}(t) \simeq-\frac{\theta t}{2}+\frac{3}{8} \sqrt{\frac{\theta}{2 \pi}} G\left(t \sqrt{\frac{8 \pi}{\theta}}\right), \theta \rightarrow \infty
$$

where $G(x)$ is the Derrida-Lebowitz scaling function 55 .

In the opposite DA limit the particles in a finite system form a single cluster of $M$ particles, which move together performing the Bernoulli random walk. In this case the exact cumulant generating function is

$$
\begin{aligned}
\lim _{t \rightarrow \infty} t^{-1} \ln \left\langle e^{\gamma Y_{t}}\right\rangle & =\ln \left(1-p+p e^{\gamma M}\right) . \\
& \simeq M p \gamma+M^{2} p(1-p) \frac{\gamma^{2}}{2},
\end{aligned}
$$

where in the second line we show the two first terms of the small $\gamma$ expansion. These are the only terms responsible for the limit $\lim _{M \rightarrow \infty} M^{2}\left(\lim _{t \rightarrow \infty} t^{-1} \ln \left\langle e^{\gamma Y_{t}}\right\rangle-M p \gamma\right)=M^{4} \gamma^{2}$ to exist under condition $\gamma M^{2}=$ const. This agrees with the behavior of (60) at small $\theta$, which follows from the limiting form of function $\mathcal{G}_{\theta}(t)$ :

$$
\mathcal{G}_{\theta}(t) \simeq \frac{t^{2}}{2}-\frac{\theta^{2} t}{8}, \quad \theta \rightarrow 0 .
$$

The distribution of the time-averaged number of particle jumps corresponding to 60 has the form

$$
\operatorname{Prob}\left(Y_{t} / t<y\right) \sim \exp \left[-\frac{p(1-p) t}{N^{2}} \widehat{\mathcal{G}}_{\theta}\left(\frac{y-M p}{\rho p(1-p)}\right)\right],
$$

where LDF $\widehat{\mathcal{G}}_{\theta}(x)$ is the Legendre transform of $\mathcal{G}_{\theta}(t)$. The presence of the factor $t N^{-2}$ is specific for the diffusive systems, though the LDF has a nontrivial form, unlike purely quadratic Gaussian single particle case. It follows from the above analysis of the limiting behavior of $\mathcal{G}_{\theta}(t)$ that the LDF $\widehat{\mathcal{G}}_{\theta}(x)$ continuously interpolates between the Gaussian quadratic and the KPZ scaling function as $\theta$ varies from zero to infinity.

Differentiating $\Lambda(\gamma)$ we obtain the cumulants of this distribution. The first one is

$$
J \simeq M p-p(1-p) \rho \frac{\theta}{2} \frac{I_{2}(\theta)}{I_{1}(\theta)},
$$

i.e. a finite number of macroscopic clusters present on the lattice for a finite fraction of time results in a finite correction to the total number of jumps, which exactly equals to $M p$ in the DA limit. In the small- $\theta$ limit this fraction is approximately $\theta^{2} / 2$. Making a small- $\theta$ expansion one can see that this contribution, $p(1-p) \rho \theta^{2} / 8$, is indeed of the same order also depending on particle density.

The one-particle diffusion coefficient obtained from the second cumulant,

$$
\Delta=p(1-p)\left[\frac{I_{1}(2 \theta)}{I_{1}^{2}(\theta)}\left(\frac{I_{2}(2 \theta)}{I_{1}(2 \theta)}-\frac{I_{2}(\theta)}{I_{1}(\theta)}\right)\right],
$$

is finite in the thermodynamic limit, similarly to the oneparticle random walk, when it exactly equals $p(1-p)$. In the KPZ-DA transition regime, this value is corrected by the factor in the square brackets. In the limit $\theta \rightarrow 0$ this factor saturates to one, recovering the free-particle diffusion coefficient. As $\theta \rightarrow \infty$ the diffusion coefficient behaves as $\Delta \simeq(3 p(1-p) / 4) \sqrt{\pi / \theta}$ indicating the transition to KPZ behavior. What is different from the DA limit as well as from the KPZ regime is the behavior of the higher cumulants. As follows from the formula 60 they have the scaling $c_{n} \sim N^{2(n-1)}$ unlike $c_{n} \sim N^{3 / 2(n-1)}$ in the $\mathrm{KPZ}$ regime and $c_{n} \sim N^{n}$ in the DA limit. It is remarkable that in the transition regime the order of growth of 


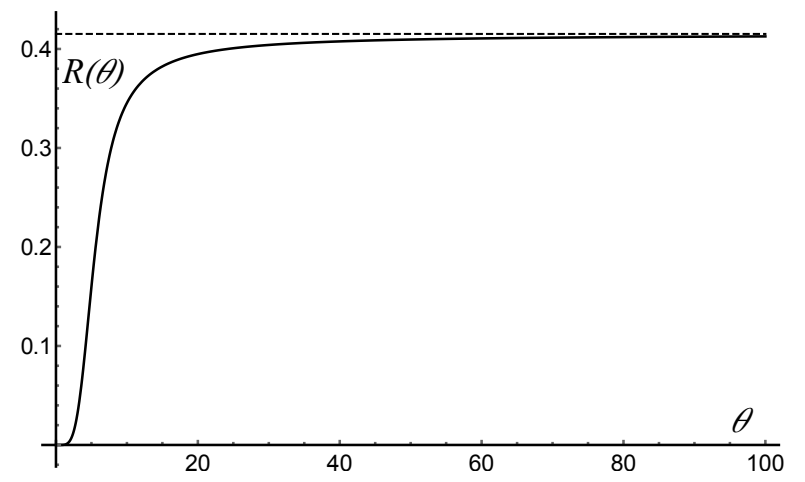

Figure 3: Universal cumulant ratio $R(\theta)$. The dashed line shows the limiting KPZ value $R(\infty)$.

the cumulants with $N$ is higher than in both the KPZ and the DA limit for $n \geq 3$. Then, it is natural to expect that the cumulants, rescaled to remove the dependence on the system size and all the other parameters except $\theta$,

$$
\tilde{c}_{n}=\lim _{N \rightarrow \infty} c_{n} \times\left[N^{2(n-1)} \rho^{n} p(1-p)\right]^{-1}=\mathcal{G}_{\theta}^{(n)}(0)
$$

will vanish in both $\theta \rightarrow 0$ and $\theta \rightarrow \infty$ limits having an extrema at some finite values of $\theta$. Indeed, as seen from Fig. 2 the third and fourth rescaled cumulants show the non-monotonous behavior having minimum and maximum at some finite values of $\theta$, respectively. The quantity, which can be used as a measure of proximity to the $\mathrm{KPZ}$ regime, is the universal cumulant ratio,

$$
R(\theta)=\frac{c_{3}^{2}}{c_{2} c_{4}}=\frac{\left(\mathcal{G}_{\theta}^{(3)}(0)\right)^{2}}{\mathcal{G}_{\theta}^{\prime \prime}(0) \mathcal{G}_{\theta}^{(4)}(0)},
$$

depending solely on the parameter $\theta$. As shown in Fig. 3. starting from zero at $\theta=0$ the ratio $R(\theta)$ monotonously approaches its limiting universal KPZ value

$$
\lim _{\theta \rightarrow \infty} R(\theta)=\frac{2\left(3 / 2-8 / 3^{3 / 2}\right)^{2}}{15 / 2-24 / \sqrt{3}+9 / \sqrt{2}} \simeq 0.41517,
$$

first obtained in 43 .

\section{UNIVERSALITY AND RELATION TO KPZ EQUATION}

In context of stochastic models, the concept of universality suggests that in the scaling limit a large class of models is characterized by probability distributions having the same universal functional form. The notion of the scaling limit implies that the temporal and spacial coordinates as well as the random variables of interest are measured in scales related to each other via simple power laws. Their exponents, usually referred to as critical exponents, is a fixed set of numbers specifying given universality class. In this way the scales are defined up to non-universal constants, which depend on parameters of the specific model. Correspondingly, numerical quantities characterizing the random variables, e.g., cumulants or correlators, depend on these constants only. As applied to the problem of KPZ interface growth in one-dimensional system of size $L$, the distribution of the height $h(x, t)$ of growing interface being a random function of the spatial and temporal coordinates $x$ and $t$ is characterized by two sets of amplitudes [35,

$$
\begin{aligned}
& a_{n}=\lim _{t \rightarrow \infty} \lim _{L \rightarrow \infty} t^{-n / 3}\left\langle(h(x, t)-\bar{h})^{n}\right\rangle_{c} \\
& b_{n}=\lim _{L \rightarrow \infty} \lim _{t \rightarrow \infty} L^{-n / 2}\left\langle(h(x, t)-\bar{h})^{n}\right\rangle_{c}
\end{aligned}
$$

for transient, $t \ll L^{3 / 2}$, and stationary, $t \gg L^{3 / 2}$, parts of evolution respectively, where $n \in \mathbb{N},\left\langle x^{n}\right\rangle_{c}$ is the notation for $n$-th cumulant of the random variable $x$, and $\bar{h}=$ $L^{-1} \int_{0}^{L} h(x, t) d x$ is the mean interface height for a given process realization. Also one can define finite time (size) corrections to the average interface velocity as compared to the one calculated at infinite time (in infinite system),

$$
\begin{aligned}
& a_{v}=\lim _{t \rightarrow \infty} \lim _{L \rightarrow \infty} t^{2 / 3}\left(\langle\partial h / \partial t\rangle-v_{\infty}\right) \\
& b_{v}=\lim _{L \rightarrow \infty} \lim _{t \rightarrow \infty} L\left(\langle\partial h / \partial t\rangle-v_{\infty}\right),
\end{aligned}
$$

where $v_{\infty}=\lim _{t, L \rightarrow \infty}\langle\partial h / \partial t\rangle$. It was conjectured in [35] that all these quantities can be expressed in terms of only two dimensional invariants. The conjecture was first proposed based on analysis of the KPZ equation itself,

$$
\frac{\partial h}{\partial t}=\widetilde{\nu} \Delta h+\widetilde{\lambda}(\nabla h)^{2}+\eta
$$

which was the first prototypical model catching the universal features of the KPZ class. Here the notations for parameters $\widetilde{\lambda}$ and $\widetilde{\nu}$ have a tilde to keep the notations traditional for KPZ equation and distinguish them from the $\nu$ and $\lambda$ of our model. The white noise $\eta$ is fully characterized by the covariance

$$
\left\langle\eta(x, t) \eta\left(x^{\prime}, t^{\prime}\right)\right\rangle=D \delta\left(x-x^{\prime}\right) \delta\left(t-t^{\prime}\right) .
$$

For an interface described by 66 the two mentioned dimensional invariants are $\widetilde{\lambda}$ and $A=D / 2 \widetilde{\nu}$. In terms of these constants the transient amplitudes 62 and 64 are given by

$$
a_{n}=\left(|\widetilde{\lambda}| A^{2}\right)^{n / 3} \widetilde{a}_{n} \text { and } a_{v}=\left(|\widetilde{\lambda}| A^{2}\right) \widetilde{a}_{v},
$$

where $\widetilde{a}_{n}$ and $\widetilde{a}_{v}$ are universal numbers. These numbers, as known from the later development of the field, must be related to cumulants of the universal distributions like the Tracy-Widom distributions, dependent on global form (large-scale) of initial conditions. For the stationary amplitudes 63 and 65 in the system with periodic boundary conditions we have

$$
b_{2}=\frac{A}{12}, \quad b_{n}=0, n>2
$$


and

$$
b_{v}=-\frac{A \widetilde{\lambda}}{2} .
$$

Vanishing of all amplitudes $b_{n}$ except the second one is due to the Gaussian stationary height distribution of the KPZ interface. The universality conjectured in 35 suggests that for an interface belonging to the KPZ class, the amplitudes $62-(65)$ have the same dependence on $\widetilde{\lambda}$ and $A$, which can in general be defined without appealing to the KPZ equation and measured experimentally. Namely, the parameter $\widetilde{\lambda}$, related to the response of the interface velocity to introducing a small tilt $h(x, t) \rightarrow h(x, t)+\kappa x$, is defined as

$$
\widetilde{\lambda}=\frac{\partial^{2} v_{\infty}}{\partial \kappa^{2}},
$$

and the parameter $A$ is the amplitude of spacial correlation function

$$
\lim _{t \rightarrow \infty}\left\langle(h(x, t)-h(y, t))^{2}\right\rangle_{c}=A|x-y| .
$$

The above studied ASEP-like system can be related to the KPZ interface on the lattice by

$$
h_{i+1}-h_{i}=1-2 \tau_{i},
$$

where $\tau_{i}=0,1$ is the occupation number of the $i$ th site and $h_{i}$ is the interface height above the bond connecting sites $i-1$ and $i$ of the lattice, $i=1, \ldots, L$. For this mapping being consistent with the number of particles on the lattice the interface must satisfy helicoidal boundary conditions

$$
h_{i+L}=h_{i}-(L-2 M),
$$

which gives a tilt $\kappa=1-2 c$ to the interface. Then the change of the interface height $\left(h_{i}(t)-h_{i}(0)\right)$ in time is nothing but twice the number of particles that have traversed the bond $(i-1, i)$ by the time $t$. Correspondingly the limiting interface speed is twice the particle current in the ASEP-like system, $v_{\infty}=2 j^{A S E P}$, where $j^{A S E P}$ was obtained in (25). Then we have

$$
\widetilde{\lambda}=\frac{1}{2} \frac{\partial^{2} j^{A S E P}}{\partial c^{2}} .
$$

Using (37) we obtain for $i \ll j$

$$
\begin{aligned}
\left\langle\left(h_{i}-h_{j}\right)^{2}\right\rangle_{c} & =4 \sum_{i \leq k, l \leq j+1}\left(\left\langle\tau_{k} \tau_{l}\right\rangle-c^{2}\right) \\
& \simeq 4 c(1-c) \operatorname{coth}\left(\frac{1}{2 \xi}\right)|i-j| ;
\end{aligned}
$$

i.e., $A=4 c(1-c) \operatorname{coth}(1 /(2 \xi))$, where $\xi$ is the correlation length 36. The explicit expressions of $\widetilde{\lambda}$ and $A$ can be found in Appendix A. Also the finite size correction $b_{v}$ to the limiting interface velocity is twice the correction to the particle current given in 24) and (57). One confirmation of the universality is the observation that the relation 65 between $b_{v}$ and the parameters $\tilde{\lambda}$ and $A$, defined by (69) and (70) respectively, holds exactly (see appendix A.).

Another demonstration of universality can be obtained using the results of Sec. [V] Note that the amplitudes 62 - 65) characterize the form of the interface relative to its average position $\bar{h}$. At the same time the absolute value of the interface height is dominated by the position of its center of mass, which, up to the bounded initial value, is $\bar{h} \simeq 2 L^{-1} Y_{t}$. Therefore, the universal LDF obtained for $Y_{t}$ also characterizes the statistics of the motion of the center of mass of interface. On the other hand, its scaling properties are expected to be defined by the dimensionful invariants $\widetilde{\lambda}$ and $A$ solely. In particular, simple dimensional arguments together with the scaling ansatz show 44 that the variance of $\bar{h}$, related to the diffusion coefficient of a particle by $\left\langle\bar{h}^{2}\right\rangle_{c}=4 c^{2} \Delta t$, has the form

$$
\left\langle\bar{h}^{2}\right\rangle_{c}=s_{0} A^{3 / 2}|\widetilde{\lambda}| L^{-1 / 2} t,
$$

where $s_{0}=\sqrt{\pi} / 4$ is a universal number first obtained in [45]. Comparing this formula with the expression (58) obtained for $\Delta$, we obtain relation A10 between $b, A$, and the density $c$, which is indeed confirmed from explicit calculations in Appendix A. The same arguments can be applied to the cumulants of an arbitrary order. In general, all the model dependence of the scaled cumulant generating function (and hence of the LDF) obtained in Sec. $[\mathrm{IV}$ is incorporated into two constants $a$ and $b$. It takes some algebra to show that these constants can be reexpressed in terms of the dimensional invariants $A$ and $\widetilde{\lambda}$ of this section:

$$
\begin{aligned}
a & =\frac{\sqrt{2 A}|\tilde{\lambda}|(1-c)^{3 / 2}}{4 \sqrt{\pi}}, \\
b & =-\operatorname{sgn} \tilde{\lambda} \frac{\sqrt{\pi A / 2}}{(1-c)^{3 / 2}} .
\end{aligned}
$$

In the spirit of universality we conjecture this relation to be universal. Up to our knowledge, it did not yet explicitly appear in the literature.

Finally it is informative to see how the system approaches the DA limit. As $\lambda \rightarrow \infty$, we asymptotically have

$$
A \simeq 8 \sqrt{\lambda}[c(1-c)]^{3 / 2} \text { and } \tilde{\lambda} \simeq-\frac{3(1-p) p}{8(1-c)^{5 / 2} c^{1 / 2}} \sqrt{\frac{1}{\lambda}} .
$$

As we saw, the KPZ regime (in particular, the universal scaling form of the LDF of interface height) holds until the value of $\lambda$ becomes of the order of $\lambda \sim N^{2}$, i.e., $A$ and $\widetilde{\lambda}$ being of order $L$ and $1 / L$, respectively. Remarkably the product $\widetilde{\lambda} A$ proportional to $b_{v}$, which is related to the typical fluctuation range, stays finite in the limit 
$\lambda \rightarrow \infty$. Therefore, first, up to the scale $\lambda \sim N^{2}$, the increase of $\lambda$ affects only non-universal constants preserving the universal functional form (54) of the LDF. Then, in the scale $\lambda \sim N^{2}$ the functional form of LDF starts to gradually change until reaching the purely Gaussian form.

\section{Acknowledgments}

We acknowledge the financial support from the Government of the Russian Federation within the framework of the implementation of the 5-100 Programme Roadmap of the National Research University Higher School of Economics. The work was also supported by the RFBR grant under Project 14-01-00474-a and by Heisenberg-Landau program.

\section{Appendix A: Explicit expressions of model-dependent constants and universal relations}

Though the scaling functions found from the asymptotic analysis are of a rather simple form, the modeldependent constants expressed as functions of particle density are very cumbersome. It is much more efficient to consider them as functions of associated fugacities. In particular, the fugacity $z_{-}$appears in the analysis of the stationary measure of the ZRP-like system in Sec. IIIB as the saddle point, where the main contribution to the integrals comes from. Its relation to the particle density in the TASEP-like system is given in 22 , or conversely

$$
c=\frac{(1-\nu) z_{-}}{1-\nu\left(2-z_{-}\right) z_{-}} .
$$

Then we obtain the mean number of particle jumps per unit time,

$$
\begin{aligned}
J & =N \frac{z_{-}(\mu-\nu)}{\left(1-\mu z_{-}\right)\left(1-\nu z_{-}\right)} \\
& +\frac{(1-\mu)(\mu-\nu)}{(1-\nu)} \frac{\left(1-z_{-}\right) z_{-}\left(1-\nu z_{-}\right)\left(1-\mu \nu z_{-}^{3}\right)}{\left(1-\mu z_{-}\right)^{3}\left(1-\nu z_{-}^{2}\right)^{2}},
\end{aligned}
$$

which being divided by size of the system $L$, yields the thermodynamic value of the mean particle current,

$$
\begin{aligned}
j_{\infty} & =(1-c) \frac{z_{-}(\mu-\nu)}{\left(1-\mu z_{-}\right)\left(1-\nu z_{-}\right)} \\
& =\frac{(\mu-\nu)\left(1-z_{-}\right) z_{-}}{\left(1-\mu z_{-}\right)\left(1-\nu\left(2-z_{-}\right) z_{-}\right)},
\end{aligned}
$$

plus the $1 / L$ finite-size correction

$$
\begin{aligned}
L\left(j_{L}-j_{\infty}\right) & =\frac{(1-\mu)(\mu-\nu)}{(1-\nu)} \\
\times & \frac{\left(1-z_{-}\right) z_{-}\left(1-\nu z_{-}\right)\left(1-\mu \nu z_{-}^{3}\right)}{\left(1-\mu z_{-}\right)^{3}\left(1-\nu z_{-}^{2}\right)^{2}} .
\end{aligned}
$$

These expressions show up again in Sec. IVB, where the latter one appears to be a product of the two modeldependent constants,

$$
\begin{aligned}
a & =\frac{(1-\mu)(\mu-\nu)}{\sqrt{2 \pi}(1-\nu)^{3 / 2}} \\
& \times \frac{\sqrt{z_{-}}\left(1-z_{-}\right)^{2}\left(1-\nu z_{-}\right)^{2}\left(1-\mu \nu z_{-}^{3}\right)}{\left(1-\mu z_{-}\right)^{3}\left(1-\nu z_{-}^{2}\right)^{5 / 2}}, \\
b & =\frac{\sqrt{2 \pi(1-\nu) z_{-}\left(1-\nu z_{-}^{2}\right)}}{\left(1-z_{-}\right)\left(1-\nu z_{-}\right)},
\end{aligned}
$$

which also determine the scaling behavior of all higher cumulants, such as diffusion coefficient, as well as the characteristic temporal and fluctuation scales. Indeed, multiplying A5 and A6 we obtain exactly A4 confirming the announced relation (57).

Another fugacity $z^{*}$, given in (35), appears in Sec. IIIC from transfer-matrix analysis of the TASEP-like system. The two seemingly unrelated sets of results obtained from ZRP-like and TASEP-like systems turn out to be linked, when one checks the universal relations between the two-dimensional invariants obtained in KPZ theory. One of the invariants, the amplitude of the correlation function $\sqrt{70}$, is given in terms of $z^{*}$ by

$$
\begin{aligned}
A & =4 c(1-c) \frac{\lambda_{1}+\lambda_{2}}{\lambda_{1}-\lambda_{2}} \\
& =\frac{4(1-\nu) z^{*}\left(z^{*}+1\right)}{\left(\left(z^{*}+1\right)^{2}-4 \nu z^{*}\right)^{3 / 2}},
\end{aligned}
$$

while the other one, the non-linearity coefficient from KPZ equation, obtained from the second derivative of particle current depending on $z_{-}$:

$$
\begin{aligned}
\widetilde{\lambda} & =\frac{1}{2} \frac{\partial^{2} j_{\infty}}{\partial c^{2}}=\frac{1}{2}\left(\frac{1}{\partial c / \partial z_{-}} \frac{\partial}{\partial z_{-}}\right)^{2} j_{\infty} \\
& =-\frac{(1-\mu)(\mu-\nu)}{(1-\nu)^{2}} \frac{\left(1-\nu\left(2-z_{-}\right) z_{-}\right)^{3}\left(1-\mu \nu z_{-}^{3}\right)}{\left(1-\mu z_{-}\right)^{3}\left(1-\nu z_{-}^{2}\right)^{3}} .
\end{aligned}
$$

Noting that the fugacities are related by

$$
z^{*}=\frac{z_{-}\left(\nu z_{-}-1\right)}{z_{-}-1}
$$

we find that the product of the dimensional invariants is equal to

$$
\widetilde{\lambda} A=-2 b_{v},
$$

where $b_{v}=2 a b=2 L\left(j_{L}-j_{\infty}\right)$, which is nothing but the relation (65). Another relation,

$$
b=\frac{\sqrt{\pi A}}{\sqrt{2}(1-c)^{3 / 2}},
$$


can be verified by direct examining formulas A1 A6 A7 A9 . This complies with another prediction of KPZ theory 71 in conjunction with the formula (58) obtained for the diffusion coefficient. Then the connection 7273 between $a, b$ and $\widetilde{\lambda}, A$ is straightforward.

\section{Appendix B: Hypergeometric functions}

\section{Gauss hypergeometric functions}

Series representation:

$$
{ }_{2} F_{1}(a, b ; c ; x)=\sum_{n=0}^{\infty} \frac{(a)_{n}(b)_{n}}{(c)_{n} n !} x^{n}
$$

Generating function for terminating series ${ }_{2} F_{1}(a, b ; c ; x)$ with $a$ negative integer:

$$
\begin{aligned}
G(x, t) & \equiv \frac{(1-x t)^{\alpha}}{(1-t)^{\beta}} \\
& =\sum_{n=0}^{\infty} \frac{(\beta)_{n}}{n !}{ }_{2} F_{1}(-n,-\alpha,-\beta-n+1 ; x) t^{n}
\end{aligned}
$$

Euler transformation:

$$
F(a, b ; c ; z)=(1-z)^{c-a-b} F(c-a, c-b ; c ; z)
$$

Chu-Vandermonde identity:

$$
{ }_{2} F_{1}(-n,-\alpha,-\beta-n+1 ; 1)=\frac{(\beta-\alpha)_{n}}{(\beta)_{n}}
$$

\section{Appell hypergeometric function $F_{1}$}

Series representations:

$$
F_{1}\left(\alpha ; \beta, \beta^{\prime} ; \gamma ; x, y\right)=\sum_{n, m=0}^{\infty} \frac{(\alpha)_{m+n}(\beta)_{m}\left(\beta^{\prime}\right)_{n}}{(\gamma)_{m+n} m ! n !} x^{m} y^{n}
$$

Generating function for terminating series $F_{1}\left(\alpha ; \beta, \beta^{\prime} ; \gamma ; x, y\right)$ with $\alpha$ negative integer :

$$
\begin{aligned}
G(x, y, z) & \equiv(1-z)^{\alpha}(1-x z)^{-\beta}(1-y z)^{-\beta^{\prime}} \\
& =\sum_{n=0}^{\infty} \frac{(-\alpha)_{n}}{n !} F_{1}\left(-n ; \beta, \beta^{\prime} ; \alpha-n+1 ; x, y\right) z^{n}
\end{aligned}
$$

One-variable reduction:

$$
F_{1}\left(\alpha ; \beta, \beta^{\prime} ; \gamma ; x, 0\right)={ }_{2} F_{1}(\alpha, \beta ; \gamma ; x)
$$

Generalized Chu-Vandermonde identity

$$
F_{1}\left(-n ; \beta, \beta^{\prime} ; \alpha-n+1 ; x, 1\right)=\frac{\left(\beta^{\prime}-\alpha\right)_{n}}{(-\alpha)_{n}} F_{1}\left(-n, \beta ; \alpha-\beta^{\prime}-n+1 ; x\right)
$$

\section{Appendix C: Modified Bessel function}

Integral representation:

$$
I_{k}(y)=\int_{0}^{2 \pi} \exp (y \cos \varphi+k i \varphi) \frac{d \varphi}{2 \pi}
$$

Asymptotic behavior:

$$
\begin{aligned}
& I_{\alpha}(x)=\frac{e^{x}}{\sqrt{2 \pi x}}\left(1-\frac{4 \alpha^{2}-1}{8 x}\right), x \rightarrow \infty \\
& I_{\alpha}(x)=\frac{1}{\alpha !}\left(\frac{x}{2}\right)^{\alpha}, x \rightarrow 0
\end{aligned}
$$

[1] H. Spohn, Large Scale Dynamics of Interacting Particles, (Springer, Berlin, 1991).

[2] T.L. Liggett, Stochastic interacting systems: contact, voter and exclusion processes, (Springer, Berlin, 1999)

[3] G.M. Schütz, Solvable models for many-body systems far from equilibrium. In C.Domb and J.Lebowitz (eds.) 
Phase Transitions and Critical Phenomena, Vol.19 (Academic, London, pp.1-251, 2001).

[4] S.F. Edwards and D.R. Wilkinson, Proc. R. Soc. A 381,17 (1982)

[5] M. Kardar, G. Parisi, and Y. C. Zhang, Phys. Rev. Lett. 56, 889 (1986)

[6] L. H. Gwa and H. Spohn Phys. Rev. A 46, 844 (1992)

[7] D. Kim, Phys. Rev. E 52, 3512 (1995)

[8] B. Derrida and J.L. Lebowitz, Phys. Rev. Lett. 80, 209 (1998)

[9] J. de Gier and F. H. L. Essler , Phys. Rev. Lett. 107, 010602. (2011)

[10] M. Gorissen, A. Lazarescu, K. Mallick, and C. Vanderzande, Phys. Rev. Lett. 109, 170601 (2012)

[11] G.M. Schütz, J. Stat. Phys. 88, 427 (1997)

[12] V. B. Priezzhev, Phys. Rev. Lett. 91, 050601 (2003)

[13] T. Sasamoto, J. Phys. A 38, L549 (2005)

[14] A. Borodin, P.L. Ferrari, M. Prähofer, and T. Sasamoto, J. Stat. Phys. 1291055 (2007)

[15] T. Imamura and T. Sasamoto, J. Stat. Phys. 128 799-846 (2007)

[16] A. Borodin, P.L. Ferrari, M. Prähofer, and T. Sasamoto, Int. Math. Res. Papers, rpm002 (2007)

[17] A. Borodin and P.L. Ferrari, Electron. J. Probab. 13, 1380 (2008)

[18] A. Borodin, P.L. Ferrari and T. Sasamoto, Comm. Pure Appl. Math. 61, 1603 (2008)

[19] A. M. Povolotsky, V. B. Priezzhev and G. M. Schütz, J. Stat. Phys. 142, 754 (2011)

[20] S. S. Poghosyan, A. M. Povolotsky, and V. B. Priezzhev, J. Stat. Mech. 08 P08013 (2012)

[21] J.G. Brankov, V.B. Priezzhev and R.V. Shelest, Phys. Rev. E 69066136 (2004)

[22] A.M. Povolotsky and V.B. Priezzhev, J. Stat. Mech. P07002 (2006)

[23] S. S. Poghosyan, V. B. Priezzhev, and G. M. Schütz, J. Stat. Mech. P04022 (2010)
[24] M. Woelki, Steady States of discrete mass transport models, master thesis, University of Duisburg-Essen (2005)

[25] A.E. Derbyshev, S.S. Poghosyan, A.M. Povolotsky, and V.B. Priezzhev, J. Stat. Mech. P05014 (2012)

[26] A.M. Povolotsky, J. Phys. A 46, 465205 (2013)

[27] I. Corwin, Int. Math. Res. Notices rnu094 (2014)

[28] A. Borodin, I. Corwin, L. Petrov, and T. Sasamoto, arXiv:1407.8534

[29] A.M. Povolotsky and J. F. F. Mendes, J. Stat. Phys. 123, $125(2006)$

[30] D. S. Lee and D. Kim, Phys. Rev. E 59, 6476 (1999)

[31] A. M. Povolotsky, V. B. Priezzhev, and C. K. Hu, J. Stat. Phys. 111, 1149 (2003)

[32] A. M. Povolotsky, Phys. Rev. E 69, 061109 (2004)

[33] T. Antal and G.M. Schütz, Phys. Rev. E 62, 83 (2000)

[34] S.N. Majumdar, S.Krishnamurthy, and M. Barma, Phys. Rev. Lett. 81, 3691 (1998)

[35] J. Krug, P. Meakin, and T. Halpin-Healy, Phys. Rev. A 45, 638 (1992)

[36] M.R. Evans, S. N. Majumdar, and R.K.P. Zia, J. Phys. A 37, L275 (2004)

[37] M. R. Evans, Braz. J. Phys. 3042 (2000)

[38] P. Bialas, Z. Burda, and D. Johnston, Nucl. Phys. B 493 505 (1997)

[39] J. G. Brankov, V.V. Papoyan, V. S., Poghosyan, and V.B. Priezzhev, Physica A 368, 471 (2006)

[40] M. Kanai, J. Phys. A 407127 (2007)

[41] M. Schreckenberg, A. Schadschneider, K. Nagel, and N. Ito, Phys. Rev. E 51, 2939 (1995)

[42] N. Rajewsky, L. Santen, A. Schadschneider and M. Schreckenberg J. Stat. Phys. 92, 151 (1998)

[43] B. Derrida, C. Appert, J. Stat. Phys. 94, 1 (1999)

[44] J. Krug, Adv. in Phys. 46, 139 (1997)

[45] B. Derrida, M.R. Evans, and D. Mukamel, J. Phys. A 26, 4911 (1993) 